\title{
Optimal bundle formation and pricing of two products with limited stock
}

\author{
Ülkü Gürler, Salih Öztop, Alper Şen* \\ Department of Industrial Engineering, Bilkent University, Bilkent, 06800 Ankara, Turkey
}

\section{A R T I C L E I N F O}

\section{Article history:}

Received 17 July 2006

Accepted 14 November 2008

Available online 24 December 2008

\section{Keywords:}

Revenue management

Pricing

Product bundling

\begin{abstract}
A B S T R A C T
In this study, we consider the stochastic modeling of a retail firm that sells two types of perishable products in a single period not only as independent items but also as a bundle. Our emphasis is on understanding the bundling practices on the inventory and pricing decisions of the firm. One of the issues we address is to decide on the number of bundles to be formed from the initial product inventory levels and the price of the bundle to maximize the expected profit. Product demands follow a Poisson Process with a price dependent rate. Customer reservation prices are assumed to have a joint distribution. We study the impact of reservation price distributions, initial inventory levels, product prices, demand arrival rates and cost of bundling. We observe that the expected profit decreases as the correlation between the reservation prices of two products increases. With negative correlation, bundling cost has a significant impact on the number of bundles formed. When the product prices are low, the retailer sells individual products as well as the bundle (mixed bundling), when they are high, the retailer sells only bundles (pure bundling). The expected profit and the number of bundles offered decrease as the variance of the reservation price distribution increases. For high starting inventory levels, the retailer reduces bundle price and offers more bundles. The number of bundle sales decreases and the number of individual product sales increases when the arrival rate increases since the need for bundling decreases. Impacts of substitutability and complementarity of products are also investigated. The retailer forms more bundles, or charges higher prices for the bundle or both as the products become more complementary and less substitutable.
\end{abstract}

(c) 2009 Elsevier B.V. All rights reserved.

\section{Introduction}

Product bundling is the practice of package selling of several goods, and offers the opportunity to reduce costs and thereby to achieve greater economic efficiency. In recent years, product bundling has gained more importance since the firms are moving toward the provision of integrated solutions as a demand stimulating strategy and a device for more competitive power, that consist of

\footnotetext{
* Corresponding author. Fax: +90312 2664054.

E-mail addresses: ulku@bilkent.edu.tr (Ü. Gürler), salih@bilkent.edu.tr (S. Öztop), alpersen@bilkent.edu.tr (A. Șen).
}

services and products sold in a bundle. Significant savings are reported for the giant consumer products and packaged goods company Procter \& Gamble through an approach called expressive competition that involves lowest-price reverse auctions and package biddings, where product bundling (attaching Crest toothpaste to a bottle of Scope mouthwash or a bundle of Crest toothbrush with its toothpaste) is used effectively throughout the process (Sandholm et al., 2006). Apart from the retail environment mentioned above, companies practice bundling in a broad range of industries including information goods (e.g., software such as Microsoft's Office Suite), travel services (e.g., vacation packages from travel agencies), restaurants (e.g., McDonald's Happy Meal), 
durable consumer goods (e.g., personal computer options), and non durable consumer goods (e.g., dishwasher detergent and rinse aid packages). While we are only focusing on pricing efficiencies obtained through bundling, bundles are offered for a variety of other reasons. Strategically, a company may use bundling to preserve (or increase) market power or to extend its market power in one product to another. Furthermore, for a firm with a broad line of products, product bundling can be used as a price strategy alternative to the more traditional follow-the-leader and cost-based strategies (Adams and Yellen, 1976; Guiltinan, 1987; Bakos and Brynjolfsson, 1999). Efficiency reasons include achieving cost savings and quality improvements. See Nalebuff (2003) for a detailed recent discussion of the motivations behind bundling practices.

Revenue management deals with the sales of perishable products in a finite horizon by controlling price and inventory with the objective of increasing revenue. In this paper, we study product bundling in revenue management. Specifically, we consider a retailer that sells a limited stock of two products facing random demand over a finite selling horizon. We focus on the inventory level of the bundle and the pricing decisions of the retailer. Hence, the portion of the initial inventory of the two products that will be used to form bundles is also a decision in our model.

The implementation of bundling may require a number of important and rather difficult decisions. First, the benefits of bundling need to be quantified in order to see whether these benefits justify the potential costs and additional complexity in operations. Also, if the company is offering more than two products, it needs to specify the number of different bundle types to offer and what products to include in each specific bundle. For products that are sold as part of a bundle, the company also needs to decide whether it will continue to sell these products individually (i.e., mixed bundling) or not (i.e., pure bundling). Finally, the company needs to determine the bundle prices and individual product prices that will maximize its profits.

Our focus in this paper is product bundling as opposed to price bundling. For the difference, we refer the reader to Stremersch and Tellis (2002) who define price bundling as "the sale of two or more separate products in a package at a discount, without any integration of products" and product bundling as "the integration and sale of two or more separate products or services at any price". Since usually physical integration needs to take place before the demand for product bundles, bundle formation decisions, i.e., how many units of individual products should be converted to bundles, are also crucial for product bundling.

Previous research on bundling in marketing and economics literature attempts to identify demand settings for which bundling is profitable. The purchase behavior of the customers is usually characterized by the reservation price (maximum price a customer is willing to pay for a product) distributions of the products. Correlation between the reservation prices, complementarity, substitutability, and heterogeneity of valuations among customers are major factors studied in this literature. The earliest study to address such issues is by Stigler (1963) who assumes additive reservation prices for the bundle and concludes that the profitability of bundling is due to the negative correlation in reservation prices. Adams and Yellen (1976) use the same settings and argue that the profitability of bundling can stem from its ability to sort customers into groups with different reservation price characteristics, and hence, extract consumer surplus. Considering the three bundling strategies, unbundling, pure bundling and mixed bundling, they conclude that relative profitability of these three strategies depend on the distribution of the reservation prices and the structure of the costs (see also Jeuland, 1984). In numerous experiments they have provided, it is found that some form of bundling is more profitable than simple monopoly pricing and bundling seems to be a more efficient method than price discrimination. Schmalensee (1984) modifies the framework of Stigler (1963) by assuming bivariate normal reservation price distribution and allowing for positive correlation. He shows that pure bundling operates by reducing the effective dispersion in buyers' tastes, since the standard deviation of reservation prices for the bundle is less than the sum of the standard deviations for the two components as long as reservation prices are not perfectly correlated. Schmalensee (1984) also shows that mixed bundling combines the advantages of pure bundling and unbundling strategies. This policy enables the seller to reduce effective heterogeneity among those buyers with high reservation prices for both goods, while still selling at a high markup to those buyers willing to pay a high price for only one of the goods. In a comment to Schmalensee (1984), Long (1984) relaxes the normality assumption on reservation price distributions and also concludes that the most favorable case for bundling as a price discrimination device is when the bundle components have negatively correlated reservation prices. Focusing on graphical analysis of bundling, Salinger (1995) indicates that if bundling does not lower costs, it tends to be profitable with negatively correlated reservation prices that are high relative to costs. If bundling lowers costs and costs are high relative to reservation values, positively correlated reservation values increase the incentive to bundle.

Although not directly related to our study, see also Ansari et al. (1996) for the determination of the optimal number of items to be included in a service bundle, Ben-Akiva and Gershenfeld (1998) for customer choice behavior for bundles with correlated demand, Carbajo et al. (1990) for incentives for bundling under imperfect competition, Hanson and Martin (1990) for the calculation of optimal bundle prices in a deterministic setting, and Stremersch and Tellis (2002) for a clear discussion of bundling terms which are used in marketing, economics, and law literature in a somewhat unclear way. Finally, we note the growing literature on bundling of information goods (see for example, Bakos and Brynjolfsson, 1999). However, the setting for the information goods is distinctly different from physical goods and most services, since the marginal costs are close to zero and inventory is almost never a constraint. 
The basic assumption in the related marketing and economics literature is that there is an abundant supply of the products, perhaps at a certain cost. Since supply is not a constraint, there is also no distinction between price bundling and product bundling (except perhaps when the reservation price of a product bundle is greater than the sum of individual product reservation prices). In many industries, however, the supply process is rather inflexible and slow. For example, in fashion retailing, for a majority of the items, there is a very limited replenishment opportunity within the short fashion season and the pre-season orders constitute a big portion of the total orders (Sen, 2008). Therefore, procurement and initial pricing decisions are often interdependent (see Choi, 2007; Karakul, 2008 for two examples) and further pricing decisions has to take the remaining inventory into account. In this study, we assume that a one time procurement decision is already made and that there is an initial inventory of items that is to be sold over a finite horizon. Therefore our approach is in line with the approach taken in the revenue management literature. See Talluri and van Ryzin (2004) for a detailed review of revenue management research and Elmaghraby and Keskinocak (2003) for a review of dynamic pricing research and practice in this context.

The papers that could be considered most directly related to our work in the revenue management literature are those studying multiple product revenue management problems as introduced in Gallego and van Ryzin (1997). Netessine et al. (2004) study a problem where they consider an e-commerce seller that dynamically forms and prices product or service packages. The problem is modeled as a dynamic program based on two possibilities in case of stock-out: an emergency replenishment of the customer's initial request or lost sales. Our model differs from Netessine et al. (2004) as we assume posted prices and product bundles and we explicitly model the consumer choice given that she is given three alternatives upfront: either one of the products or the bundle or none. Ernst and Kouvelis (1999) study a newsboy type modeling framework where the retail firm sell products not only as independent items but also as part of a bundle (or a packaged good). They study a problem similar to ours, however they focus on the inventory decisions only, taking the price set as a given parameter. They also examine the switching between the individual products and the packaged good when there are stock-outs. Through a numerical study, they show that positive correlation of original demands favors increased stocking levels of a multi-product package, while negative correlation tends to have the opposite effect. They also show that correlated demands result in higher profitability and stronger substitutability results in higher stocking levels for the packaged good.

Bulut et al. (2009) study the single period pricing of two perishable products which are sold individually and as a bundle. Their customer demand has a Poisson distribution with a price dependent arrival rate. Assuming a general reservation price distribution, they determine the optimal product prices that maximize the expected revenue. They also compare the performance of different bundling strategies under different conditions such as different reservation price distributions, demand arrival rates, and starting inventory levels. Their numerical study demonstrates that, when individual product prices are fixed to high values, the expected revenue is a decreasing function of the correlation coefficient, while for low prices the expected revenue is an increasing function of the correlation coefficient. They indicate that, bundling is least effective in case of limited supply and the mixed bundling strategy outperforms the others, especially when the customer reservation prices are negatively correlated. The main differences between this work and ours is that (i) we assume product bundles rather than price bundles and thus the initial inventory level of bundles is a decision variable. Hence the inventory dynamics progress as if there are three substitutable products, whereas in Bulut et al. (2009), a bundle can not be offered to the customer unless both products are available; (ii) we allow for customer switches to other products in case of stock-outs at the end of the horizon. This takes into account the dynamic behavior of the customer and needs to incorporate switching probabilities explicitly to the analysis. As such, although the present work is related to theirs, the different assumptions mentioned above call for a separate analysis that can not be deduced from their work.

\subsection{Scope of our study and summary of results}

We consider a retail firm that sells two types of perishable products over a finite selling season. The starting inventory levels of these two products are fixed and at the beginning of the season, the retailer uses all or a portion of these initial stocks to form product bundles. The retailer then sells the bundles as well as the individual products (mixed bundling) by charging constant prices over the selling season. We determine the optimal number of bundles that the retailer should form and the optimal individual and bundle prices that the retailer should charge so as to maximize his expected profit over the selling season. No replenishments are allowed during the selling season, and separation of bundles into individual items is not allowed. We also investigate the effect of cost of forming bundles, as these costs could be non negligible in some industries. For example, combining separate PC components into a PC requires technicians to work on, which adds a labor cost to bundling (see Ansari et al., 1996 for a model to determine the number of bundles to be formed).

Customer arrivals follow a Poisson process with a constant arrival rate and their choice of individual products or the product bundle is governed by their reservation prices. "Posted" product prices are used which means prices are known by the customers, however they do not know the available inventory before they actually arrive at the store. When they arrive, if their preferred product is not available, they may switch to another product or do not make a purchase. These switching probabilities depend on the reservation prices and posted prices set by the retailer. 
Using a numerical study, we investigate the effects of various factors on our model, such as the correlation between the reservation price distributions, the variance of the reservation price distributions, initial inventory levels, the bundle formation cost, and the intensity of the customer arrivals. We observe that the expected profit decreases as the correlation coefficient increases and increase in bundling cost lowers the profit. With negative correlation, bundling cost has a significant impact on the number of bundles formed. However with positive correlation, this effect is negligible.

When the product prices are below the mean value of the reservation price distribution, the retailer sets a high bundle price and sells individual products as well as the bundle. When the individual products are high, the retailer charges a bundle price such that only bundles are sold. The expected profit and the optimal number of bundles formed decrease as the variance of the reservation price distribution increases. However, the optimal bundle price has different a behavior depending on the correlation of the reservation prices. For negative correlation, optimal bundle price decreases as the standard deviation increases. For positive correlation, the optimal bundle price is an increasing function of the standard deviation. For the uncorrelated case, the optimal bundle price is a decreasing function of the standard deviation for small bundling cost values and it is an increasing function for large bundling cost values.

Finally we perform numerical analysis to investigate the impact of product substitutability and complementarity. For all correlation values and bundle formation costs, we see that the retailer is forming more bundles, or charging higher prices for the bundle, or both as the products become more complementary and less substitutable. The impact of substitutability or complementarity is more pronounced when the product prices are uncorrelated and positively correlated.

The rest of the paper is organized as follows. Section 2 formulates the problem investigated and explains the stochastic model used in our study. In Section 3 we give results of our numerical studies and in the final section we conclude with the discussion of our major findings and the avenues for future research.

\section{Model and the analysis}

We consider a retailer that sells two product types (products 1 and 2) and a bundle (which is formed with the two products) over a finite selling season. Initial inventory levels, $Q_{1}$ and $Q_{2}$, of products 1 and 2 are given and the retailer decides the number of bundles to be formed with this initial inventory $\left(n_{b}\right)$ with a unit bundling cost of $c$. Once $n_{b}$, the inventory level of the bundles at the beginning of the season is decided, no new bundles are formed and none of them are unbundled to offer individual products during the season.

A customer is allowed to buy only one type of product, which means he or she can buy one unit of products 1,2 or a bundle but not any combination of the products. The customer can also leave the store without buying any product. The prices of products 1,2 and the bundle $\left(p_{1}, p_{2}\right.$ and $p_{b}$ ) are determined such that $p_{b} \leqslant p_{1}+p_{2}$. Prices are set at the beginning of the selling season and they are fixed during the period. The objective of the retailer is to maximize the expected profit over this single selling season.

Customer arrivals to the store follow a Poisson process with a fixed arrival rate of $\lambda$ per season. The decision to buy a product is determined by the comparison of the customer's reservation price with the product prices. Purchase probabilities for products 1, 2 and the bundle are denoted as $m_{1}\left(p_{1}, p_{2}, p_{b}\right), m_{2}\left(p_{1}, p_{2}, p_{b}\right)$ and $m_{b}\left(p_{1}, p_{2}, p_{b}\right)$, respectively. For brevity, we express $m_{i}\left(p_{1}, p_{2}, p_{b}\right)$ as $m_{i}$ for $i=1,2, b$. Then $m_{0}=1-m_{1}-m_{2}-$ $m_{b}$ is the probability of no purchase. The arrival rates $\ell_{1}, \ell_{2}$ for the two products and the bundle $\ell_{b}$ are then given as $\ell_{i}=\lambda \times m_{i} i=1,2, b$.

Customer reservation prices are random variables. Reservation price of product 1 is $R_{1}$ and reservation price of product 2 is $R_{2}$. Mean and variance parameters of the reservation price distribution are $\left(\mu_{1}, \sigma_{1}\right)$ and $\left(\mu_{2}, \sigma_{2}\right)$, for products 1 and 2 , respectively. We now discuss main assumptions used in our model. First, in this study we are not concerned with estimating reservation price distributions. We believe that this merits a separate study and we refer the reader to Jedidi and Zhang (2002) for an example. Our model in the first part is structured on the main assumption that reservation price for the bundle is the sum of the reservation prices of individual products that form the bundle, i.e., $R_{b}=R_{1}+R_{2}$. This is one of the common assumptions used in the bundling literature such as Adams and Yellen (1976), Schmalensee (1984) and McAffe et al. (1989). Guiltinan (1987) called this assumption as "the assumption of strict additivity". In the last part, we relax this assumption following a model in Venkatesh and Kamakura (2003) and analyze complementary products leading to superadditive reservation prices and substitutable products leading to subadditive reservation prices.

\subsection{Purchasing probabilities}

Let $f_{R_{1}, R_{2}}\left(r_{1}, r_{2}\right)$ denote the joint reservation price density for the two products. When all products are available, purchasing probabilities are calculated by comparing the customer reservation price with the product price. A customer buys either a single product or a bundle or leaves the store without buying any product. Probability expressions of these events are stated below:

Probability of no purchase: A customer will leave the store without buying any product when his reservation prices for each product and the bundle are all lower than their corresponding sales prices. Therefore, the probability of no purchase is stated as,

$$
\begin{aligned}
m_{0} & =\operatorname{Pr}\left\{R_{1} \leqslant p_{1} ; R_{2} \leqslant p_{2} ; R_{b} \leqslant p_{b}\right\} \\
& =\operatorname{Pr}\left\{R_{1} \leqslant p_{1} ; R_{2} \leqslant \min \left\{p_{2}, p_{b}-R_{1}\right\}\right. \\
& =\int_{-\infty}^{p_{1}} \int_{-\infty}^{a_{1}} f_{R_{1}, R_{2}}\left(r_{1}, r_{2}\right) \mathrm{d} r_{1} \mathrm{~d} r_{2}
\end{aligned}
$$

where $a_{1}=\min \left(\left\{p_{2}, p_{b}-r_{1}\right\}\right)$. 
Probability of purchasing product 1: Purchase probabilities are calculated by comparing the consumer surplus, which is the difference between the reservation price and the product price. A customer will purchase product 1 if his surplus from product 1 is positive and greater than his surplus values from other products. Thus the probability of purchasing product 1 is stated as,

$$
\begin{aligned}
m_{1} & =\operatorname{Pr}\left\{R_{1} \geqslant p_{1} ; R_{1}-p_{1} \geqslant R_{2}-p_{2} ; R_{1}-p_{1} \geqslant R_{b}-p_{b}\right\} \\
& =\int_{p_{1}}^{\infty} \int_{-\infty}^{a_{2}} f_{R_{1}, R_{2}}\left(r_{1}, r_{2}\right) \mathrm{d} r_{1} \mathrm{~d} r_{2}
\end{aligned}
$$

where $a_{2}=\min \left(\left\{r_{1}-p_{1}+p_{2}, p_{b}-p_{1}\right\}\right)$.

Probability of purchasing product 2: Similarly,

$$
\begin{aligned}
m_{2} & =\operatorname{Pr}\left\{R_{2} \geqslant p_{2} ; R_{2}-p_{2} \geqslant R_{1}-p_{1} ; R_{2}-p_{2} \geqslant R_{b}-p_{b}\right\} \\
& =\int_{p_{2}}^{\infty} \int_{-\infty}^{a_{3}} f_{R_{1}, R_{2}}\left(r_{1}, r_{2}\right) \mathrm{d} r_{2} \mathrm{~d} r_{1}
\end{aligned}
$$

where $a_{3}=\min \left(\left\{r_{2}-p_{2}+p_{1}, p_{b}-p_{2}\right\}\right)$.

Probability of purchasing a bundle: Again using the same reasoning,

$$
\begin{aligned}
m_{b} & =\operatorname{Pr}\left\{R_{b} \geqslant p_{b} ; R_{b}-p_{b} \geqslant R_{1}-p_{1} ; R_{b}-p_{b} \geqslant R_{2}-p_{2}\right\} \\
& =\int_{p_{b}-p_{2}}^{\infty} \int_{a_{4}}^{\infty} f_{R_{1}, R_{2}}\left(r_{1}, r_{2}\right) \mathrm{d} r_{1} \mathrm{~d} r_{2}
\end{aligned}
$$

where $a_{4}=\max \left(\left\{p_{b}-r_{1}, p_{b}-p_{1}\right\}\right)$.

\subsection{Switching probabilities}

We now consider situations when one or two products run out of stock during the selling season. We are interested in calculating the probability that a customer who had originally intended to purchase a particular product $i$ will switch to another product $j$, if product $i$ runs out of stock during the selling season. We call these switching probabilities and denote them by $\gamma_{i j}$ if only product $i$ ran out of stock and the customer potentially has two products to choose from or by $\gamma_{i j}^{-}$if two products ran out of stock and the customer only has product $j$ to switch to.

\subsubsection{One type of product incurs shortage}

Probability of switching from product 1 to bundle or to product 2: Suppose product 1 incurs shortage and the customer has the option to switch to the bundle, product 2 or to leave without purchase. Let $\gamma_{1 B}, \gamma_{12}$ and $1-\gamma_{1 B}-\gamma_{12}$ be the probability of these events, respectively. Since the switching events follow after the first choices of the customers are made, we need to calculate these probabilities conditional on the event that the first preference of the customer was to buy product 1 . Then we have

$$
\begin{aligned}
\gamma_{1 B}= & \operatorname{Pr}\left\{R_{b}-p_{b} \geqslant R_{2}-p_{2} ; R_{b} \geqslant p_{b} \mid R_{1}-p_{1} \geqslant R_{b}-p_{b} ;\right. \\
& \left.R_{1}-p_{1} \geqslant R_{2}-p_{2} ; R_{1} \geqslant p_{1}\right\} \\
= & \operatorname{Pr}\left\{R_{1} \geqslant \max \left(p_{b}-p_{2}, p_{1}\right) ; \min \left(p_{b}-p_{1}, R_{1}-p_{1}+p_{2}\right)\right. \\
& \left.\geqslant R_{2} \geqslant p_{b}-R_{1}\right\} / m_{1} \\
= & \operatorname{Pr}\left\{R_{1} \geqslant \max \left(p_{b}-p_{2}, p_{1}\right) ; p_{b}-p_{1} \geqslant R_{2} \geqslant p_{b}-R_{1}\right\} / m_{1} \\
= & \int_{\max \left(p_{b}-p_{2}, p_{1}\right)}^{\infty} \int_{p_{b}-r_{1}}^{p_{b}-p_{1}} f_{R_{1}, R_{2}}\left(r_{1}, r_{2}\right) \mathrm{d} r_{1} \mathrm{~d} r_{2} / m_{1} .
\end{aligned}
$$

Similarly we have

$$
\begin{aligned}
\gamma_{12}= & \operatorname{Pr}\left\{R_{2}-p_{2} \geqslant R_{b}-p_{b} ; R_{2} \geqslant p_{2} \mid R_{1}-p_{1} \geqslant R_{b}-p_{b} ;\right. \\
& \left.R_{1}-p_{1} \geqslant R_{2}-p_{2} ; R_{1} \geqslant p_{1}\right\} \\
= & \int_{p_{1}}^{p_{b}-p_{2}} \int_{p_{2}}^{p_{2}+r_{1}-p_{1}} f_{R_{1}, R_{2}}\left(r_{1}, r_{2}\right) \mathrm{d} r_{1} \mathrm{~d} r_{2} / m_{1} .
\end{aligned}
$$

Probability of switching from product 2 to bundle or to product 1: Similar to the previous case let $\gamma_{2 B}, \gamma_{21}$ be the probability of switching to a bundle or to product 1 when product 2 stocks out. We have

$$
\begin{aligned}
\gamma_{2 B}= & \operatorname{Pr}\left\{R_{b}-p_{b} \geqslant R_{1}-p_{1} ; R_{b} \geqslant p_{b} \mid R_{2}-p_{2} \geqslant R_{b}-p_{b} ;\right. \\
& \left.R_{2}-p_{2} \geqslant R_{1}-p_{1} ; R_{2} \geqslant p_{2}\right\} \\
= & \int_{\max \left(p_{b}-p_{1}, p_{2}\right)}^{\infty} \int_{p_{b}-r_{2}}^{p_{b}-p_{2}} f_{R_{1}, R_{2}}\left(r_{1}, r_{2}\right) \mathrm{d} r_{2} \mathrm{~d} r_{1} / m_{2}
\end{aligned}
$$

and

$$
\begin{aligned}
\gamma_{21}= & \operatorname{Pr}\left\{R_{1}-p_{1} \geqslant R_{b}-p_{b} ; R_{1} \geqslant p_{1} \mid R_{2}-p_{2} \geqslant R_{b}-p_{b} ;\right. \\
& \left.R_{2}-p_{2} \geqslant R_{1}-p_{1} ; R_{2} \geqslant p_{2}\right\} \\
= & \int_{p_{2}}^{p_{b}-p_{1}} \int_{p_{1}}^{r_{2}-p_{2}+p_{1}} f_{R_{1}, R_{2}}\left(r_{1}, r_{2}\right) \mathrm{d} r_{2} \mathrm{~d} r_{1} / m_{2} .
\end{aligned}
$$

Probability of switching from bundle to product 1 or to product 2: Now let $\gamma_{B 1}$ and $\gamma_{B 2}$ be the probability of switching to product 1 or to product 2 when bundle stocks our. We have

$$
\begin{aligned}
\gamma_{B 1}= & \operatorname{Pr}\left\{R_{1}-p_{1} \geqslant R_{2}-p_{2} ; R_{1} \geqslant p_{1} \mid R_{b}-p_{b} \geqslant R_{1}-p_{1} ;\right. \\
& \left.R_{b}-p_{b} \geqslant R_{2}-p_{2} ; R_{b} \geqslant p_{b}\right\} \\
= & \int_{\max \left(p_{1}, p_{b}-p_{2}\right)}^{\infty} \int_{p_{b}-p_{1}}^{r_{1}-p_{1}+p_{2}} f_{R_{1}, R_{2}}\left(r_{1}, r_{2}\right) \mathrm{d} r_{1} \mathrm{~d} r_{2} / m_{b}
\end{aligned}
$$

and

$$
\begin{aligned}
\gamma_{B 2}= & \operatorname{Pr}\left\{R_{2}-p_{2} \geqslant R_{1}-p_{1} ; R_{2} \geqslant p_{2} \mid R_{b}-p_{b} \geqslant R_{1}-p_{1} ;\right. \\
& \left.R_{b}-p_{b} \geqslant R_{2}-p_{2} ; R_{b} \geqslant p_{b}\right\} \\
= & \int_{\max \left(p_{2}, p_{b}-p_{1}\right)}^{\infty} \int_{p_{b}-p_{2}}^{r_{2}-p_{2}+p_{1}} f_{R_{1}, R_{2}}\left(r_{1}, r_{2}\right) \mathrm{d} r_{2} \mathrm{~d} r_{1} / m_{b} .
\end{aligned}
$$

\subsubsection{Two types of products incur shortage}

Products 1 and 2 incur shortage: Let $\gamma_{1 B}^{-}, \gamma_{2 B}^{-}$be the probability of switching from product 1 or 2 to bundle when only bundle is available. Then we have,

$$
\begin{aligned}
\gamma_{1 B}^{-}= & \operatorname{Pr}\left\{R_{b} \geqslant p_{b} \mid R_{1}-p_{1} \geqslant R_{b}-p_{b} ; R_{1}-p_{1} \geqslant R_{2}-p_{2} ; R_{1} \geqslant p_{1}\right\} \\
= & \operatorname{Pr}\left\{R_{1}+R_{2} \geqslant p_{b} ; R_{1}-p_{1} \geqslant R_{1}+R_{2}-p_{b} ;\right. \\
& \left.R_{1}-p_{1} \geqslant R_{2}-p_{2} ; R_{1} \geqslant p_{1}\right\} / m_{1} \\
= & \operatorname{Pr}\left\{R_{1} \geqslant p_{1} ; \min \left(p_{b}-p_{1}, R_{1}-p_{1}+p_{2}\right) \geqslant R_{2} \geqslant p_{b}-R_{1}\right\} / m_{1} \\
= & \int_{p_{1}}^{\infty} \int_{p_{b}-r_{1}}^{\min \left(p_{b}-p_{1}, r_{1}-p_{1}+p_{2}\right)} f_{R_{1}, R_{2}}\left(r_{1}, r_{2}\right) \mathrm{d} r_{1} \mathrm{dr} r_{2} / m_{1}
\end{aligned}
$$

and

$$
\begin{aligned}
\gamma_{2 B}^{-} & =\operatorname{Pr}\left\{R_{b} \geqslant p_{b} \mid R_{2}-p_{2} \geqslant R_{b}-p_{b} ; R_{2}-p_{2} \geqslant R_{1}-p_{1} ; R_{2} \geqslant p_{2}\right\} \\
& =\int_{p_{2}}^{\infty} \int_{p_{b}-r_{2}}^{\min \left(r_{2}-p_{2}+p_{1}, p_{b}-p_{2}\right)} f_{R_{1}, R_{2}}\left(r_{1}, r_{2}\right) \mathrm{d} r_{2} \mathrm{~d} r_{1} / m_{2} .
\end{aligned}
$$

Bundle and product 2 incur shortage: Let $\gamma_{B 1}^{-}, \gamma_{21}^{-}$ be the probability of switching from bundle or products 2 to 1 when only product 1 is available. 
Then

$$
\begin{aligned}
\gamma_{B 1}^{-} & =\operatorname{Pr}\left\{R_{1} \geqslant p_{1} \mid R_{b}-p_{b} \geqslant R_{1}-p_{1} ; R_{b}-p_{b} \geqslant R_{2}-p_{2} ; R_{b} \geqslant p_{b}\right\} \\
& =\int_{\max \left(p_{1}, p_{b}-p_{2}\right)}^{\infty} \int_{p_{b}-p_{1}}^{\infty} f_{R_{1}, R_{2}}\left(r_{1}, r_{2}\right) \mathrm{d} r_{1} \mathrm{~d} r_{2} / m_{b} .
\end{aligned}
$$

and we have

$$
\begin{aligned}
\gamma_{21}^{-} & =\operatorname{Pr}\left\{R_{1} \geqslant p_{1} \mid R_{2}-p_{2} \geqslant R_{b}-p_{b} ; R_{2}-p_{2} \geqslant R_{1}-p_{1} ; R_{2} \geqslant p_{2}\right\} \\
& =\int_{p_{1}}^{p_{b}-p_{2}} \int_{r_{1}-p_{1}+p_{2}}^{\infty} f_{R_{1}, R_{2}}\left(r_{1}, r_{2}\right) \mathrm{d} r_{1} \mathrm{~d} r_{2} / m_{2}
\end{aligned}
$$

Bundle and product 1 incur shortage: Similar to the previous case let $\gamma_{B 2}^{-}$and $\gamma_{12}^{-}$be the probability of switching when only product 2 is available. Then,

$$
\begin{aligned}
\gamma_{B 2}^{-} & =\operatorname{Pr}\left\{R_{2} \geqslant p_{2} \mid R_{b}-p_{b} \geqslant R_{1}-p_{1} ; R_{b}-p_{b} \geqslant R_{2}-p_{2} ; R_{b} \geqslant p_{b}\right\} \\
& =\int_{p_{b}-p_{2}}^{\infty} \int_{\max \left(p_{2}, p_{b}-p_{1}\right)}^{\infty} f_{R_{1}, R_{2}}\left(r_{1}, r_{2}\right) \mathrm{d} r_{1} \mathrm{~d} r_{2} / m_{b}
\end{aligned}
$$

and

$$
\begin{aligned}
\gamma_{12}^{-} & =\operatorname{Pr}\left\{R_{2} \geqslant p_{2} \mid R_{1}-p_{1} \geqslant R_{b}-p_{b} ; R_{1}-p_{1} \geqslant R_{2}-p_{2} ; R_{1} \geqslant p_{1}\right\} \\
& =\int_{p_{2}}^{p_{b}-p_{1}} \int_{r_{2}-p_{2}+p_{1}}^{\infty} f_{R_{1}, R_{2}}\left(r_{1}, r_{2}\right) \mathrm{d} r_{2} \mathrm{~d} r_{1} / m_{1} .
\end{aligned}
$$

\subsection{Sales probabilities and the objective function}

Recall that $Q_{1}$ and $Q_{2}$ are the initial inventory levels of products 1 and 2 , respectively. Let $n_{b}$ be the number of bundles formed (or the starting inventory level of the bundle) and $n_{i}, i=1,2$ be the remaining units of product $i$, with $n_{1}=Q_{1}-n_{b}$ and $n_{2}=Q_{2}-n_{b}$.

Also let $X_{1}, X_{2}$, and $X_{b}$ denote the number of customers whose first preference are for products 1,2 , and the bundle, respectively. We also have the random variables corresponding to the number of customers that switch from one product to another. These variables are denoted as $X_{i j}$, where $i$ is the customer initial preference and $j$ is the type of the product that the customer switches to (substitutes for $i$ ).

The realized values of these random variables will be denoted by $x_{1}, x_{2}, x_{b}, x_{1 b}, x_{12}, x_{2 b}, x_{21}, x_{b 1}$ and $x_{b 2}$. Let

$P\left\{\begin{array}{c}x_{1}, x_{2}, x_{b}, x_{1 b}, x_{12}, \\ x_{2 b}, x_{21}, x_{b 1}, x_{b 2}\end{array}\right\}=P\left\{\begin{array}{c}X_{1}=x_{1}, X_{2}=x_{2}, X_{b}=x_{b}, \\ X_{1 b}=x_{1 b}, X_{12}=x_{12}, X_{2 b}=x_{2 b}, \\ X_{21}=x_{21}, X_{b 1}=x_{b 1}, X_{b 2}=x_{b 2}\end{array}\right\}$

denote the joint probability mass function of those random variables. Note that for certain realizations we only need joint marginal probability mass function of only a subset of these variables.

When all dedicated demand can be satisfied, due to the independence property of the Poisson processes, we have

$$
\begin{aligned}
\operatorname{Pr}\left\{X_{1}=\right. & \left.x_{1}, X_{2}=x_{2}, X_{b}=x_{b}\right\}=\operatorname{Pr}\left\{X_{1}=x_{1}\right\} \operatorname{Pr}\left\{X_{2}=x_{2}\right\} \\
& \times \operatorname{Pr}\left\{X_{b}=x_{b}\right\}
\end{aligned}
$$

where $X_{i}$ has a Poisson distribution with rate $\ell_{i}=\lambda \times m_{i}$, $i=1,2, b$.

For the derivation of the expected profit, $\pi$, there are eight possible realizations when only the initial choices are considered. After this first classification, sub-cases are defined to include the switching customer realizations. All realization cases are listed in Table 1.

We have two further assumptions regarding the allocation of inventory and customer switches during the stock-out situations, to simplify the analysis. First, we assume that the inventory of a product (or the bundle) is first allocated to customers whose first choice is that product (or the bundle). The customers that cannot find their first choices switch to their second choice (if their surplus is also positive for the second choice) and the demands resulting from these switches are satisfied with the remaining inventory of their second choice.

For these cases we assume that switching behavior will follow a Multinomial distribution since there are three possible choices. However, if the second choice also runs out of stock, we assume that there are no further switches. Then the customer has the alternatives to switch to the available product or to leave without any purchase; therefore binomial distribution is used to calculate the switching probabilities. We now will provide the expressions of these realizations. Due to space limitations we only provide the details of Cases 1, 2, 5 and 8. Cases 3 and 4 can be derived similar to Case 2. Cases 6 and 7 can be derived similar to Case 5.

Case 1: No shortage occurs $\left(x_{1} \leqslant n_{1}, x_{2} \leqslant n_{2}, x_{b} \leqslant n_{b}\right)$. Expected profit in the region where all customers are satisfied by their first choice products is given by $\pi_{1}$

Table 1

Cases of realizations to derive the expected profit.

Case 1: No shortage occurs

Case 2: Bundle incurs shortage

(a) All excess demand of the bundle is satisfied

(b) Product 1 incurs shortage with the excess bundle demand

(c) Product 2 incurs shortage with the excess bundle demand

(d) Both products incur shortage with the excess bundle demand

Case 3: Product 1 incurs shortage

(a) All excess demand of product 1 is satisfied

(b) Product 2 incurs shortage with the excess demand of product 1

(c) Bundle incurs shortage with the excess demand of product 1

(d) Product 2 and bundle incur shortage with the excess demand of product 1

Case 4: Product 2 incurs shortage

(a) All excess demand of product 2 is satisfied

(b) Product 1 incurs shortage with the excess demand of product 2

(c) Bundle incurs shortage with the excess demand of product 2

(d) Product 1 and bundle incur shortage with the excess demand of product 2

Case 5: Product 1 and the bundle incur shortage

(a) All excess demand of product 1 and the bundle are satisfied

(b) Product 2 incurs shortage with the excess demand of product 1 and the bundle

Case 6: Product 2 and the bundle incur shortage

(a) All excess demand of product 2 and the bundle are satisfied

(b) Product 1 incurs shortage with the excess demand of product 2 and the bundle

Case 7: Products 1 and 2 incur shortage

(a) All excess demand of the products are satisfied from the bundle

(b) Bundle incurs shortage with the excess demand of two products

Case 8: All products incur shortage 
which is

$$
\begin{aligned}
\pi_{1}= & \sum_{x_{1}=0}^{n_{1}} \sum_{x_{2}=0}^{n_{2}} \sum_{x_{b}=0}^{n_{b}}\left(p_{1} x_{1}+p_{2} x_{2}+p_{b} x_{b}-c n_{b}\right) P\left(x_{1}, x_{2}, x_{b}\right) \\
= & \sum_{x_{1}=0}^{n_{1}} \sum_{x_{2}=0}^{n_{2}} \sum_{x_{b}=0}^{n_{b}}\left(p_{1} x_{1}+p_{2} x_{2}+p_{b} x_{b}-c n_{b}\right) \\
& \times \frac{\mathrm{e}^{-\ell_{1}} \ell_{1}^{x_{1}}}{x_{1} !} \frac{\mathrm{e}^{-\ell_{2}} \ell_{2}^{x_{2}}}{x_{2} !} \frac{\mathrm{e}^{-\ell_{b}} \ell_{b}^{x_{b}}}{x_{b} !} .
\end{aligned}
$$

Case 2: Bundle incurs shortage $\left(x_{1} \leqslant n_{1}, x_{2} \leqslant n_{2}, x_{b}>n_{b}\right)$. Initial demand for the bundle is more than the available stock but initial demands for products 1 and 2 are satisfied from the stocks. Excess demand of the bundle customers can result in four sub-cases.

(a) All excess demand of the bundle is satisfied. Let $x_{b}-$ $n_{b}$ be the number of excess bundle customers. In this case, $x_{b}-n_{b}$ units are satisfied from the excess inventories of products 1 and 2 . That is, we have $x_{1}+x_{b 1} \leqslant n_{1}$, $x_{2}+x_{b 2} \leqslant n_{2}, x_{1} \leqslant n_{1}, x_{2} \leqslant n_{2}, x_{b}>n_{b}$ and the contribution of this case to the total expected profit is given by

$$
\begin{aligned}
\pi_{2 a}= & \sum_{x_{1}=0}^{n_{1}} \sum_{x_{2}=0}^{n_{2}} \sum_{x_{b}=n_{b}+1}^{\infty} \sum_{x_{b 1}=0}^{x_{b}-n_{b}} \sum_{x_{b 2}=0}^{x_{b}-n_{b}-x_{b 1}} \\
& \times\left(p_{1}\left(x_{1}+x_{b 1}\right)+p_{2}\left(x_{2}+x_{b 2}\right)+p_{b} n_{b}-c n_{b}\right) \\
& \times \frac{\mathrm{e}^{-\ell_{1}} \ell_{1}^{x_{1}}}{x_{1} !} \frac{\mathrm{e}^{-\ell_{2}} \ell_{2}^{x_{2}}}{x_{2} !} \frac{\mathrm{e}^{-\ell_{b}} \ell_{b}^{x_{b}}}{x_{b} !} \frac{\left(x_{b}-n_{b}\right) !}{x_{b 1} ! x_{b 2} ! x_{b 0} !}\left(\gamma_{B 1}\right)^{x_{b 1}}\left(\gamma_{B 2}\right)^{x_{b 2}}\left(\gamma_{B 0}\right)^{x_{b 0}}
\end{aligned}
$$

where $x_{b 0}=x_{b}-n_{b}-x_{b 1}-x_{b 2}$ and $\gamma_{B 0}=1-\gamma_{B 1}-\gamma_{B 2}$.

(b) Product 1 incurs shortage with the excess bundle demand. In this case, original demand for product 1 is satisfied but the left over is not sufficient to satisfy the overflow from the bundle customers. All demands for product 2 are satisfied from the stock. For this case we have $x_{1}+x_{b 1}>n_{1}, x_{2}+x_{b 2} \leqslant n_{2}, x_{1} \leqslant n_{1}, x_{2} \leqslant n_{2}, x_{b}>n_{b}$ and the contribution is given by

$$
\begin{aligned}
\pi_{2 b}= & \sum_{x_{1}=0}^{n_{1}} \sum_{x_{2}=0}^{n_{2}} \sum_{x_{b}=n_{b}+1}^{\infty} \sum_{x_{b 1}=n_{1}+1-x_{1}}^{x_{b}-n_{b}} \sum_{x_{b 2}=0}^{x_{b}-n_{b}-x_{b 1}} \\
& \left(p_{1} n_{1}+p_{2}\left(x_{2}+x_{b 2}\right)+p_{b} n_{b}-c n_{b}\right) \\
& \times \frac{\mathrm{e}^{-\ell_{1}} \ell_{1}^{x_{1}}}{x_{1} !} \frac{\mathrm{e}^{-\ell_{2}} \ell_{2}^{x_{2}}}{x_{2} !} \frac{\mathrm{e}^{-\ell_{b}} \ell_{b}^{x_{b}}}{x_{b} !} \frac{\left(x_{b}-n_{b}\right) !}{x_{b 1} ! x_{b 2} ! x_{b 0} !} \\
& \times\left(\gamma_{B 1}\right)^{x_{b 1}}\left(\gamma_{B 2}\right)^{x_{b 2}}\left(\gamma_{B 0}\right)^{x_{b 0}} .
\end{aligned}
$$

(c) Product 2 incurs shortage with the excess bundle demand. This case is similar to the above case, except that product 2 incurs shortage. Hence we have $x_{1}+x_{b 1} \leqslant n_{1}$, $x_{2}+x_{b 2}>n_{2}, x_{1} \leqslant n_{1}, x_{2} \leqslant n_{2}, x_{b}>n_{b}$ and the contribution is given by

$$
\begin{aligned}
\pi_{2 c}= & \sum_{x_{1}=0}^{n_{1}} \sum_{x_{2}=0}^{n_{2}} \sum_{x_{b}=n_{b}+1}^{\infty} \sum_{x_{b 1}=0}^{x_{b}-n_{b}} \sum_{x_{b 2}=n_{2}+1-x_{2}}^{x_{b}-n_{b}-x_{b 1}} \\
& \left(p_{1}\left(x_{1}+x_{b 1}\right)+p_{2} n_{2}+p_{b} n_{b}-c n_{b}\right) \\
& \times \frac{\mathrm{e}^{-\ell_{1}} e_{1}^{x_{1}}}{x_{1} !} \frac{\mathrm{e}^{-\ell_{2}} \ell_{2}^{x_{2}}}{x_{2} !} \frac{\mathrm{e}^{-\ell_{b}} \ell_{b}^{x_{b}}}{x_{b} !} \frac{\left(x_{b}-n_{b}\right) !}{x_{b 1} ! x_{b 2} ! x_{b 0} !} \\
& \times\left(\gamma_{B 1}\right)^{x_{b 1}}\left(\gamma_{B 2}\right)^{x_{b 2}}\left(\gamma_{B 0}\right)^{x_{b 0}} .
\end{aligned}
$$

(d) Both products incur shortage with the excess bundle demand. In this case the excess inventories of products 1 and 2 are not sufficient to satisfy the overflow demand from the bundle customers. That is, we have $x_{1}+x_{b 1}>n_{1}, x_{2}+x_{b 2}>n_{2}, x_{1} \leqslant n_{1}, x_{2} \leqslant n_{2}, x_{b}>n_{b}$ and

$$
\begin{aligned}
\pi_{2 d}= & \sum_{x_{1}=0}^{n_{1}} \sum_{x_{2}=0}^{n_{2}} \sum_{x_{b}=n_{b}+1}^{\infty} \sum_{x_{b 1}=n_{1}+1-x_{1}}^{x_{b}-n_{b}} \sum_{x_{b 2}=n_{2}+1-x_{2}}^{x_{b}-n_{b}-x_{b 1}} \\
& \left(p_{1} n_{1}+p_{2} n_{2}+p_{b} n_{b}-c n_{b}\right) \\
& \times \frac{\mathrm{e}^{-\ell_{1}} \ell_{1}^{x_{1}}}{x_{1} !} \frac{\mathrm{e}^{-\ell_{2}} \ell_{2}^{x_{2}}}{x_{2} !} \frac{\mathrm{e}^{-\ell_{b}} \ell_{b}^{x_{b}}}{x_{b} !} \frac{\left(x_{b}-n_{b}\right) !}{x_{b 1} ! x_{b 2} ! x_{b 0} !} \\
& \times\left(\gamma_{B 1}\right)^{x_{b 1}}\left(\gamma_{B 2}\right)^{x_{b 2}}\left(\gamma_{B 0}\right)^{x_{b 0}} .
\end{aligned}
$$

Expected profit for the Case 2 is calculated as $\pi_{2}=$ $\pi_{2 a}+\pi_{2 b}+\pi_{2 c}+\pi_{2 d}$.

Case 5: Product 1 and bundle incur shortage $\left(x_{1}>n_{1}\right.$, $\left.x_{2} \leqslant n_{2}, x_{b}>n_{b}\right)$. Initial demands for product 1 and the bundle are greater than the respective stock amounts and only initial demand for product 2 is satisfied from the stock. Excess demands of product 1 and the bundle result in two sub-cases.

(a) All excess demand of product 1 and the bundle are satisfied. Demand for product 2 is satisfied including the switching customers from product 1 and the bundle. That is, we have $x_{2}+x_{12}+x_{b 2} \leqslant n_{2}, x_{1}>n_{1}, x_{2} \leqslant n_{2}, x_{b}>n_{b}$ and

$$
\begin{aligned}
\pi_{5 a}= & \sum_{x_{1}=n_{1}+1}^{\infty} \sum_{x_{2}=0}^{n_{2}} \sum_{x_{b}=n_{b}+1}^{\infty} \sum_{x_{12}=0}^{x_{1}-n_{1}} \sum_{x_{b 2}=0}^{x_{b}-n_{b}} \\
& \left(p_{1} n_{1}+p_{2}\left(x_{2}+x_{12}+x_{b 2}\right)+p_{b} n_{b}-c n_{b}\right) \\
& \times \frac{\mathrm{e}^{-\ell_{1}} \ell_{1}^{x_{1}} \mathrm{e}^{-\ell_{2}} \ell_{2}^{x_{2}} \mathrm{e}^{-\ell_{b}} \ell_{b}^{x_{b}}}{x_{1} ! x_{2} ! x_{b} !}\left(\begin{array}{c}
x_{1}-n_{1}+x_{12}-1 \\
x_{1}-n_{1}-1
\end{array}\right) \\
& \times\left(\gamma_{12}^{-}\right)^{x_{1}-n_{1}}\left(1-\gamma_{12}^{-}\right)^{x_{12}} \\
& \times\left(\begin{array}{c}
x_{b}-n_{b}+x_{b 2}-1 \\
x_{b}-n_{b}-1
\end{array}\right)\left(\gamma_{B 2}^{-}\right)^{x_{b}-n_{b}}\left(1-\gamma_{B 2}^{-}\right)^{x_{b 2}} .
\end{aligned}
$$

(b) Product 2 incurs shortage with the excess demand of product 1 and bundle. Initial product 2 demand is satisfied but excess demand from product 1 and bundle cannot be satisfied with the product 2 stock. We have $x_{2}+x_{12}+x_{b 2}>n_{2}, x_{1}>n_{1}, x_{2} \leqslant n_{2}, x_{b}>n_{b}$ and

$$
\begin{aligned}
\pi_{5 b}= & \sum_{x_{1}=n_{1}+1}^{\infty} \sum_{x_{2}=0}^{n_{2}} \sum_{x_{b}=n_{b}+1}^{\infty}\left(p_{1} n_{1}+p_{2} n_{2}+p_{b} n_{b}-c n_{b}\right) \\
& \sum_{x_{12}, x_{b 2}: x_{12}+x_{b 2}>n_{2}-x_{2}}^{\mathrm{e}^{-\ell_{1}} \ell_{1}^{x_{1}}} \\
& \times \frac{\mathrm{e}^{-\ell_{2}} \ell_{2}^{x_{2}}}{x_{1} !} \frac{\mathrm{e}^{-\ell_{b}} \ell_{b}^{x_{b}}}{x_{b} !}\left(\begin{array}{c}
x_{1}-n_{1}+x_{12}-1 \\
x_{1}-n_{1}-1
\end{array}\right) \\
& \times\left(\gamma_{12}^{-}\right)^{x_{1}-n_{1}}\left(1-\gamma_{12}^{-}\right)^{x_{12}} \\
& \times\left(\begin{array}{c}
x_{b}-n_{b}+x_{b 2}-1 \\
x_{b}-n_{b}-1
\end{array}\right)\left(\gamma_{B 2}^{-}\right)^{x_{b}-n_{b}}\left(1-\gamma_{B 2}^{-}\right)^{x_{b 2}} .
\end{aligned}
$$

Expected profit for the Case 5 is calculated as $\pi_{5}=$ $\pi_{5 a}+\pi_{5 b}$.

Case 8: All products incur shortage. As the last case, we consider the case where all products incur shortage with the initial dedicated customer demand. That is $x_{1}>n_{1}$, $x_{2}>n_{2}, x_{b}>n_{b}$ and

$$
\begin{aligned}
\pi_{8}= & \sum_{x_{1}=n_{1}+1}^{\infty} \sum_{x_{2}=n_{2}+1}^{\infty} \sum_{x_{b}=n_{b}+1}^{\infty}\left(p_{1} n_{1}+p_{2} n_{2}+p_{b} n_{b}\right) \\
& \times \frac{\mathrm{e}^{-\ell_{1}} \ell_{1}^{x_{1}}}{x_{1} !} \frac{\mathrm{e}^{-\ell_{2}} \ell_{2}^{x_{2}}}{x_{2} !} \frac{\mathrm{e}^{-\ell_{b}} \ell_{b}^{x_{b}}}{x_{b} !}
\end{aligned}
$$


The total expected profit is calculated as adding all expected profits contributions calculated for each cases. It can be formulated as

$\pi=\sum_{i=1}^{8} \pi_{i}$

where $i$ is the index for the cases.

\subsection{Superadditivity and subadditivity of reservation prices}

The analysis so far assumes the strict additivity of customer reservation prices where reservation price for the bundle is the sum of the reservation prices of individual products that form the bundle. When the products are complements or substitutes this assumption no longer holds. In order to model substitutability or complementarity, we use the model in Venkatesh and Kamakura (2003) who refer to the degree of substitutability and complementarity as the degree of contingency, $\theta$, and define it as

$\theta=\frac{R_{b}-\left(R_{1}+R_{2}\right)}{R_{1}+R_{2}}$.

When the individual products are complements, reservation price of the bundle is superaddivite or $\theta>0$. When the individual products are substitutes, reservation price of the bundle is subadditive, or $\theta<0$. Clearly, $\theta=0$ refers to the strict additive case.

A non-zero degree of contingency changes the purchasing $\left(m_{i}\right)$ and switching $\left(\gamma_{i j}, \gamma_{i j}^{-}\right)$probability expressions since we now have to use $R_{b}=(1+\theta)\left(R_{1}+R_{2}\right)$. Purchasing and switching probability expressions for non-zero values of $\theta$ are provided in Appendix A. Note that sales probability and objective function formulations that are provided earlier are still valid for non-zero values of $\theta$.

\section{Numerical study}

In this section, we present the results of our numerical study to demonstrate the effects of various factors on the optimal bundling and pricing policies. The factors considered are: the correlation between the reservation prices of the two products, the variance of the reservation price distributions, initial inventory levels, the unit bundle formation cost, and the intensity of the customer arrivals. We also consider the effects of the degree of product complementarity and substitutability, also known as the degree of contingency.

For the numerical study, we wrote a FORTRAN code to calculate the expected profit and to determine the optimum number of bundles to be formed and the optimum bundle price for a given parameter set.

Before providing the results, we present the setup of the model used for the numerical study. The model defined in previous section contains a general continuous bivariate distribution for customer reservation prices. In literature, personal choices are modeled using distributions of the Gaussian family (Schmalensee, 1984). Hence, we also choose bivariate normal distribution to model customer reservation prices of the two products. Normal distribution is also motivated by the facts that sum of normal variates also have normal distribution and that correlation effects are easily incorporated into the model. A disadvantage of normal distribution, that it can take negative values is avoided with a high probability by using appropriate parameters.

As stated before, $R_{1}$ and $R_{2}$ denote the reservation prices of products 1 and 2 and $f_{R_{1}, R_{2}}\left(r_{1}, r_{2}\right)$ is their joint probability density function give by

$f_{R_{1}, R_{2}}\left(r_{1}, r_{2}\right)=\frac{\mathrm{e}^{-\Gamma\left(r_{1}, r_{2}\right) / 2}}{2 \pi \sigma_{1} \sigma_{2} \sqrt{1-\rho^{2}}}$

where $\rho$ is the correlation coefficient between the reservation prices and

$$
\begin{aligned}
\Gamma\left(r_{1}, r_{2}\right)= & \frac{1}{1-\rho^{2}}\left[\left(\frac{r_{1}-\mu_{1}}{\sigma_{1}}\right)^{2}-2 \rho\left(\frac{r_{1}-\mu_{1}}{\sigma_{1}}\right)\left(\frac{r_{2}-\mu_{2}}{\sigma_{2}}\right)\right. \\
& \left.+\left(\frac{r_{1}-\mu_{1}}{\sigma_{1}}\right)^{2}\right] .
\end{aligned}
$$

Then the marginal distributions of reservation prices of products 1 and 2 are also normally with parameters $\left(\mu_{1}, \sigma_{1}\right)$ and $\left(\mu_{2}, \sigma_{2}\right)$, respectively. When bundle reservation price is strictly additive, that is $R_{b}=R_{1}+R_{2}, R_{b}$ is normally distributed with mean $\mu_{b}=\mu_{1}+\mu_{2}$ and standard deviation $\sigma_{b}$ given as

$\sigma_{b}=\left(\sigma_{1}+\sigma_{2}\right) \sqrt{1-2(1-\rho) \omega(1-\omega)}$

where $\omega=\sigma_{1} /\left(\sigma_{1}+\sigma_{2}\right)$.

To emphasize the effects of factors defined above for optimal bundling, we fix the individual product prices $p_{1}$ and $p_{2}$, and optimize the number of bundles formed, $n_{b}$, and the bundle price, $p_{b}$, although the model provided in the previous section is a general one and can be used to jointly optimize all product prices. To illustrate the overall effectiveness of bundling we also provide the percent increase in the profit compared to the unbundling case, where the two products are sold individually without a bundle offer. This case can be considered as a special case of the original model where the bundle price is set to a prohibitively high value. Because the objective function is highly complicated, an analytical result is not available for its concavity in the decision variables. Hence in the numerical section we resorted to a grid search over a wide range of values. For the optimal prices, the search started at the mean reservation price and is then extended to its neighborhood. In our experiments we observed joint concavity in $n_{b}$ and $p_{b}$, which helped in numerical search.

\subsection{The base case}

A base case is described to compare and investigate the effects of the factors listed above. In the base case, the following setting is used: individual product prices, $p_{1}$ and $p_{2}$ are set to 10 , reservation price distributions are taken such that the marginals have means $\mu_{1}=\mu_{2}=10$ and standard deviations $\sigma_{1}=\sigma_{2}=2$. Degree of contingency, $\theta$ is set to zero. Initial inventory level for individual products are $Q_{1}=Q_{2}=5$ and the arrival rate, $\lambda$ is set to 10 . We search optimal bundle price within the search interval 
with increments of 0.25 and take the unit bundling cost $c$ as $0,1,2$ and 4 . The results of the base case under the correlation coefficient, $\rho$, of $-0.9,0$ and 0.9 are tabulated in Table 2. $n_{b}^{*}$ is the optimal starting inventory level of bundles and $p_{b}^{*}$ is the optimal bundle price. The fifth column is the expected profit, the sixth column is the percent improvement of bundling strategy over unbundling. The next two columns represent the expected number of products sold. The last three columns are the arrival rate of customers dedicated to each product $\left(\ell_{1}, \ell_{2}, \ell_{b}\right)$ and the arrival rate of customers who would leave the store without buying any product $\left(\ell_{0}\right)$ even when all products are available.

We also demonstrate the results of the base case in Fig. 1. In the figures, the optimal number of bundles, the optimal bundle price and the percent increase in the profit compared to unbundling case are given in parenthesis. It is observed that both the correlation coefficient and the bundling cost $c$ have significant effects on the optimal number of bundles formed, the bundle price, and the expected profit. Obviously, increase in bundling cost results in decreasing profits. With negative correlation however, optimal number of bundles is very sensitive to the bundling cost (e.g., suggesting only one bundle for the case $c=4$ ), whereas with positive correlation, the solution calls for converting all inventory into the bundle, and only when $c=4$, one unit of each product is spared for individual purchase. We observe that the optimal bundle price and the expected profit decrease as the correlation coefficient increases. This observation is common to the other experimental cases discussed below and will be elaborated more in the following discussion.

\subsection{The impact of other factors}

The effects of product prices is investigated by setting individual product prices equal $\left(p_{1}=p_{2}\right)$ with values 8,10 and 12 , representing mean and one standard deviation below and above the mean. The other parameters are same as the base case $\left(\mu_{1}=\mu_{2}=10, \sigma_{1}=\sigma_{2}=2\right.$, $\left.Q_{1}=Q_{2}=5, \lambda=10, \rho=-0.9,0,0.9, \quad c=0,1,2,4\right)$. The

Table 2

Base case.

\begin{tabular}{|c|c|c|c|c|c|c|c|c|c|c|}
\hline$\rho$ & $c$ & $n_{b}^{*}$ & $p_{b}^{*}$ & $E$ (Profit) & $\%$ & $E\left(x_{1}\right)=E\left(x_{2}\right)$ & $E\left(x_{b}\right)$ & $\ell_{1}=\ell_{2}$ & $\ell_{b}$ & $\ell_{0}$ \\
\hline-0.9 & 0 & 5 & 19.25 & 93.0645 & 17.74 & 0.00 & 4.52 & 3.34 & 2.82 & 0.50 \\
\hline-0.9 & 1 & 4 & 19.00 & 88.3828 & 11.82 & 0.76 & 3.07 & 2.97 & 3.68 & 0.38 \\
\hline-0.9 & 2 & 3 & 19.00 & 84.8695 & 7.37 & 1.50 & 2.37 & 2.97 & 3.68 & 0.38 \\
\hline-0.9 & 4 & 1 & 19.25 & 80.6290 & 2.01 & 3.12 & 0.92 & 3.34 & 2.82 & 0.50 \\
\hline 0 & 0 & 5 & 18.75 & 87.1901 & 27.27 & 0.00 & 3.55 & 1.33 & 5.13 & 2.21 \\
\hline 0 & 1 & 4 & 18.75 & 83.0791 & 21.26 & 0.77 & 3.39 & 1.33 & 5.13 & 2.21 \\
\hline 0 & 2 & 3 & 19.00 & 79.1586 & 15.54 & 1.53 & 2.73 & 1.54 & 4.61 & 2.31 \\
\hline 0 & 4 & 2 & 19.25 & 73.3638 & 7.08 & 2.21 & 1.89 & 1.77 & 4.07 & 2.39 \\
\hline 0.9 & 0 & 5 & 18.75 & 85.3171 & 55.23 & 0.00 & 4.54 & 0.06 & 6.19 & 3.68 \\
\hline 0.9 & 1 & 5 & 18.75 & 80.3171 & 46.13 & 0.00 & 4.54 & 0.06 & 6.19 & 3.68 \\
\hline 0.9 & 2 & 5 & 18.75 & 75.3171 & 37.03 & 0.00 & 4.54 & 0.06 & 6.19 & 3.68 \\
\hline 0.9 & 4 & 4 & 18.75 & 67.0220 & 21.94 & 0.59 & 3.79 & 0.06 & 6.19 & 3.68 \\
\hline
\end{tabular}

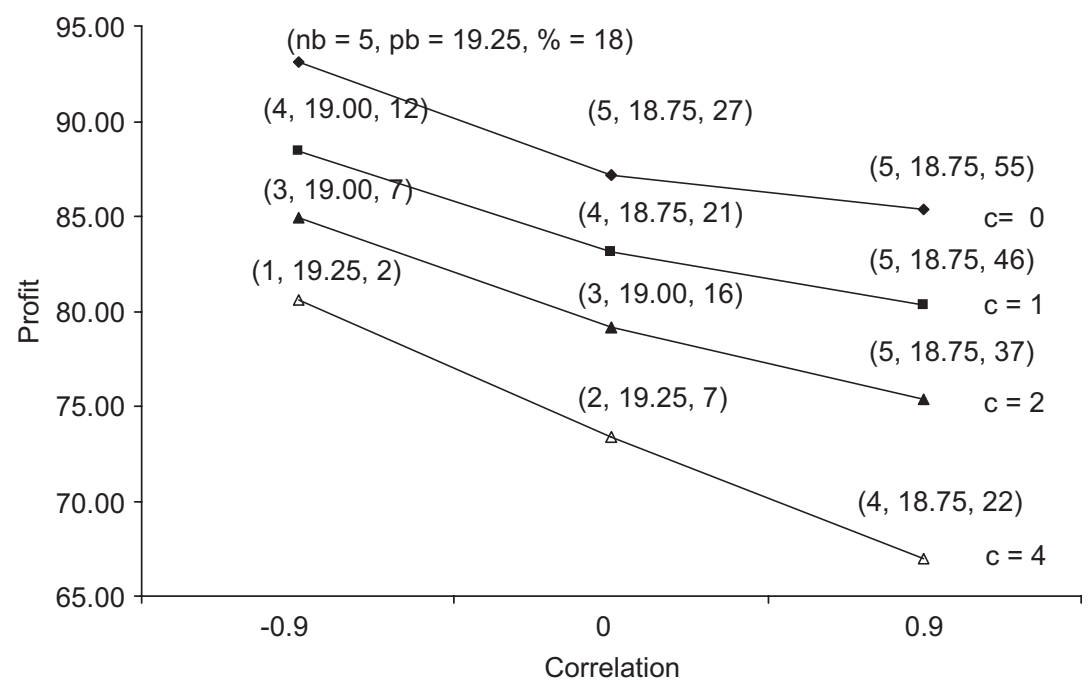

Fig. 1. Base case-profit vs. correlation. 
results are tabulated in Table 3 (this and the rest of the tables are in Appendix B) and the figures extracted from this table, Figs. 2-4 corresponding to correlation coefficient values of $-0.9,0$ and 0.9 , respectively. Figs. 2-4 show an expected concave behavior in the product prices, for all correlation levels and for almost all levels of bundle costs, but expected profit decrease significantly, as the bundling cost and correlation coefficient increases. When the product prices are low (below the mean reservation price), bundles are not desired much, i.e., mixed bundling is adopted and the optimal bundle price is the sum of individual product prices, the maximum possible price for the bundle. When the individual product prices are high, pure bundling is preferred. Maximum expected profit for high product prices is very close to the expected profits for medium prices.

The effect of initial inventory levels on the expected profit is investigated by comparing the profits for individual product inventory levels $Q_{1}=Q_{2}=5$ and 10 . The results are given in Table 4 . We observe an increase in the expected profit with the initial inventory levels, with more inventories resulting in more bundles with reduced bundle prices. This is intuitively obvious since the cost of purchasing the items is not considered and the customer arrival rate is constant. The results are particularly interesting for $\rho=-0.9$. In this case the retailer converts all inventory to the bundles regardless of the bundle formation cost. For high initial inventory levels, the optimal bundle price decreases more rapidly than the case of low initial inventory levels as the correlation coefficient increases.

Another factor we investigated is the variance of the reservation price distribution. We considered the equal standard deviation $\left(\sigma_{1}=\sigma_{2}\right)$ values of 1,2 and 3 . The results are tabulated in Table 5. Also, Figs. 5-7 demonstrate the effect of the standard deviation on the expected profit with correlation coefficient values of $-0.9,0$ and 0.9 , respectively. The obvious finding is that the expected profit decreases as the variance of the reservation price increases. For the same bundling cost value, the difference between the expected profit at $\sigma=1$ and the expected profit at $\sigma=3$ increases as the correlation coefficient increases. In other words, the effect of the variance of the reservation price on the expected profit is much more pronounced when there is a positive correlation between the reservation prices. We also note that the optimal number of bundles decreases as the standard deviation increases. Since increased variance may correspond to more 'risky' customer, and to enhance more sales, more flexibility is provided to the customer by forming less

Table 3

The impact of product prices on the expected profit: $\mu_{1}=\mu_{2}=10, \sigma_{1}=\sigma_{2}=2, \theta=0, Q_{1}=Q_{2}=5, \lambda=10$.

\begin{tabular}{|c|c|c|c|c|c|c|c|c|c|c|c|}
\hline$p_{1}=p_{2}$ & $\rho$ & $c$ & $n_{b}^{*}$ & $p_{b}^{*}$ & $E$ (Profit) & $\%$ & $E\left(x_{1}\right)=E\left(x_{2}\right)$ & $E\left(x_{b}\right)$ & $\ell_{1}=\ell_{2}$ & $\ell_{b}$ & $\ell_{0}$ \\
\hline 8 & -0.9 & 0 & 5 & 16.00 & 79.2561 & 20.18 & 0.00 & 2.81 & 1.59 & 6.83 & 0.00 \\
\hline 8 & -0.9 & 1 & 5 & 16.00 & 74.2561 & 12.60 & 0.00 & 2.81 & 1.59 & 6.83 & 0.00 \\
\hline 8 & -0.9 & 2 & 3 & 16.00 & 70.5990 & 7.06 & 1.74 & 2.84 & 1.59 & 6.83 & 0.00 \\
\hline 8 & -0.9 & 4 & 1 & 16.00 & 66.2855 & 0.52 & 3.39 & 1.00 & 1.59 & 6.83 & 0.00 \\
\hline 8 & 0 & 0 & 5 & 16.00 & 78.7881 & 21.14 & 0.00 & 3.00 & 1.33 & 7.08 & 0.25 \\
\hline 8 & 0 & 1 & 4 & 16.00 & 73.9089 & 13.64 & 0.81 & 3.44 & 1.33 & 7.08 & 0.25 \\
\hline 8 & 0 & 2 & 3 & 16.00 & 70.1810 & 7.91 & 1.75 & 2.91 & 1.33 & 7.08 & 0.25 \\
\hline 8 & 0 & 4 & 1 & 16.00 & 65.5052 & 0.72 & 3.35 & 1.00 & 1.33 & 7.08 & 0.25 \\
\hline 8 & 0.9 & 0 & 5 & 16.00 & 78.0908 & 27.20 & 0.00 & 4.38 & 0.43 & 7.98 & 1.15 \\
\hline 8 & 0.9 & 1 & 5 & 16.00 & 73.0908 & 19.05 & 0.00 & 4.38 & 0.43 & 7.98 & 1.15 \\
\hline 8 & 0.9 & 2 & 4 & 16.00 & 68.9604 & 12.32 & 0.86 & 3.92 & 0.43 & 7.98 & 1.15 \\
\hline 8 & 0.9 & 4 & 2 & 16.00 & 62.9023 & 2.46 & 2.44 & 2.00 & 0.43 & 7.98 & 1.15 \\
\hline 10 & -0.9 & 0 & 5 & 19.25 & 93.0645 & 17.74 & 0.00 & 4.52 & 3.34 & 2.82 & 0.50 \\
\hline 10 & -0.9 & 1 & 4 & 19.00 & 88.3828 & 11.82 & 0.76 & 3.07 & 2.97 & 3.68 & 0.38 \\
\hline 10 & -0.9 & 2 & 3 & 19.00 & 84.8695 & 7.37 & 1.50 & 2.37 & 2.97 & 3.68 & 0.38 \\
\hline 10 & -0.9 & 4 & 1 & 19.25 & 80.6290 & 2.01 & 3.12 & 0.92 & 3.34 & 2.82 & 0.50 \\
\hline 10 & 0 & 0 & 5 & 18.75 & 87.1901 & 27.27 & 0.00 & 3.55 & 1.33 & 5.13 & 2.21 \\
\hline 10 & 0 & 1 & 4 & 18.75 & 83.0791 & 21.26 & 0.77 & 3.39 & 1.33 & 5.13 & 2.21 \\
\hline 10 & 0 & 2 & 3 & 19.00 & 79.1586 & 15.54 & 1.53 & 2.73 & 1.54 & 4.61 & 2.31 \\
\hline 10 & 0 & 4 & 2 & 19.25 & 73.3638 & 7.08 & 2.21 & 1.89 & 1.77 & 4.07 & 2.39 \\
\hline 10 & 0.9 & 0 & 5 & 18.75 & 85.3171 & 55.23 & 0.00 & 4.54 & 0.06 & 6.19 & 3.68 \\
\hline 10 & 0.9 & 1 & 5 & 18.75 & 80.3171 & 46.13 & 0.00 & 4.54 & 0.06 & 6.19 & 3.68 \\
\hline 10 & 0.9 & 2 & 5 & 18.75 & 75.3171 & 37.03 & 0.00 & 4.54 & 0.06 & 6.19 & 3.68 \\
\hline 10 & 0.9 & 4 & 4 & 18.75 & 67.0220 & 21.94 & 0.59 & 3.79 & 0.06 & 6.19 & 3.68 \\
\hline 12 & -0.9 & 0 & 5 & 19.25 & 93.1312 & 145.78 & 0.00 & 3.89 & 0.75 & 6.86 & 1.65 \\
\hline 12 & -0.9 & 1 & 5 & 19.25 & 88.1312 & 132.58 & 0.00 & 3.89 & 0.75 & 6.86 & 1.65 \\
\hline 12 & -0.9 & 2 & 5 & 19.25 & 83.1312 & 119.39 & 0.00 & 3.89 & 0.75 & 6.86 & 1.65 \\
\hline 12 & -0.9 & 4 & 4 & 19.25 & 74.8394 & 97.51 & 0.64 & 3.79 & 0.75 & 6.86 & 1.65 \\
\hline 12 & 0 & 0 & 5 & 18.75 & 87.2226 & 149.71 & 0.00 & 4.63 & 0.08 & 6.61 & 3.22 \\
\hline 12 & 0 & 1 & 5 & 18.75 & 82.2226 & 135.39 & 0.00 & 4.63 & 0.08 & 6.61 & 3.22 \\
\hline 12 & 0 & 2 & 5 & 18.75 & 77.2226 & 121.08 & 0.00 & 4.63 & 0.08 & 6.61 & 3.22 \\
\hline 12 & 0 & 4 & 5 & 18.75 & 67.2226 & 92.45 & 0.00 & 4.63 & 0.08 & 6.61 & 3.22 \\
\hline 12 & 0.9 & 0 & 5 & 18.75 & 85.3275 & 252.66 & 0.00 & 4.55 & 0.00 & 6.26 & 3.74 \\
\hline 12 & 0.9 & 1 & 5 & 18.75 & 80.3275 & 231.99 & 0.00 & 4.55 & 0.00 & 6.26 & 3.74 \\
\hline 12 & 0.9 & 2 & 5 & 18.75 & 75.3275 & 211.33 & 0.00 & 4.55 & 0.00 & 6.26 & 3.74 \\
\hline 12 & 0.9 & 4 & 5 & 18.75 & 65.3275 & 170.00 & 0.00 & 4.55 & 0.00 & 6.26 & 3.74 \\
\hline
\end{tabular}




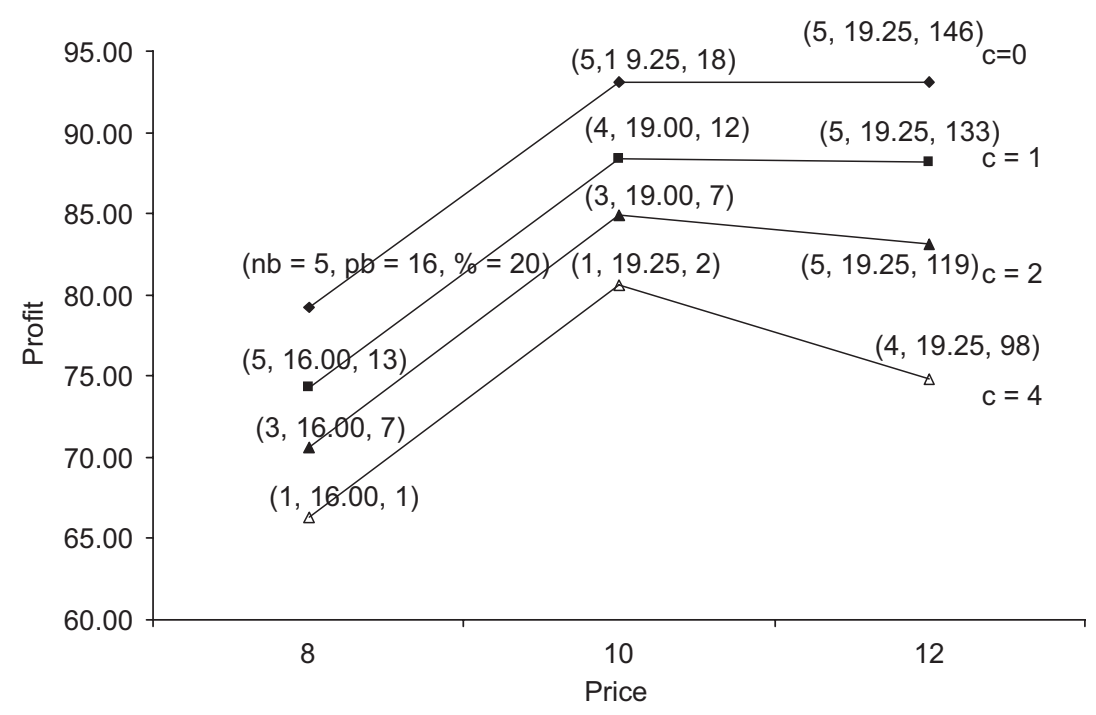

Fig. 2. Profit vs. product price for $\rho=-0.9$.

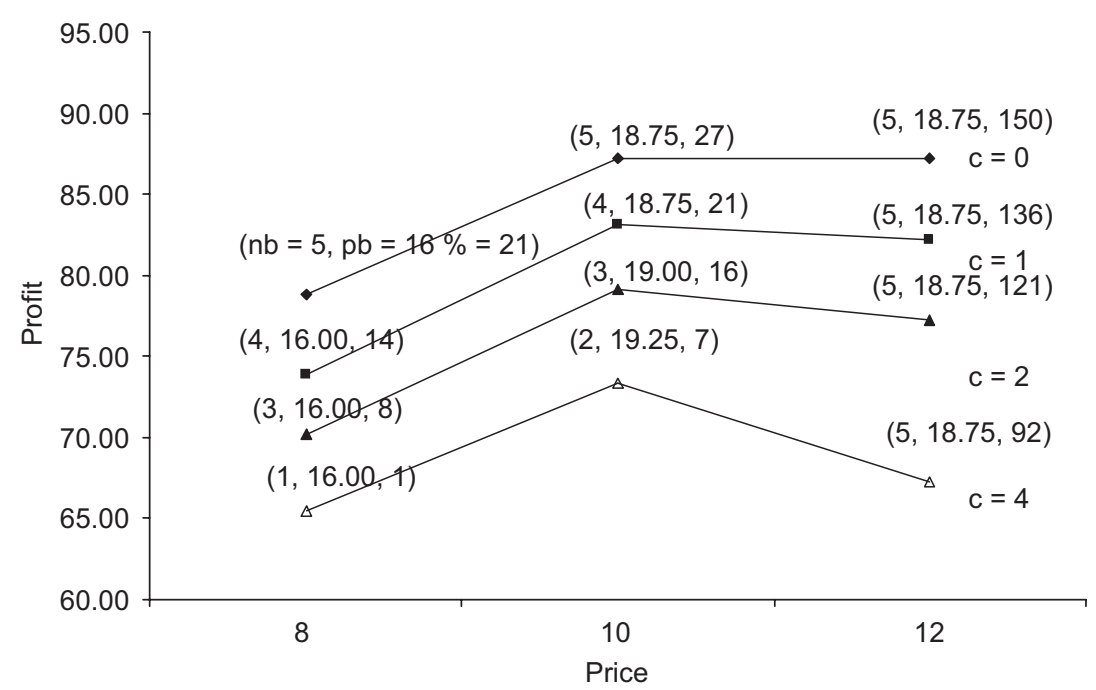

Fig. 3. Profit vs. product price for $\rho=0$.

bundles, leaving more individual products. The optimal bundle price with respect to the correlation coefficient has a different behavior, which is not immediately obvious. For negative correlation, the optimal bundle price decreases as the standard deviation increases. However for positive correlation, it is just the opposite-increasing with the standard deviation. For the zero correlation case, the bundle price decreases with the standard deviation for small bundling costs and increases in it for large bundling costs. The trend of the bundle price with respect to the variance seems to have a more complicated structure that depends also on the number bundles and the bundle cost.

Finally, we investigate the effect of the arrival rate, $\lambda$ by considering three different values 5,10 and 15 . The results are tabulated in Table 6 and Figs. 8-10. The clear finding that can be seen from Figs. 8-10 is that the expected profit is an increasing function of the arrival rate. The number of bundle sales decreases and the number of individual product sales increases when the arrival rate increases since the need for bundling decreases; the retailer can easily sell his products individually. For the negative correlation case, decrease in the optimal number of bundles formed is more significant. The retailer offers less bundles and charges higher prices for them as the arrival rate increases.

As a common observation in all the above cases, we note the significant effect of the correlation coefficient on the expected revenues and optimal bundle prices, both of 


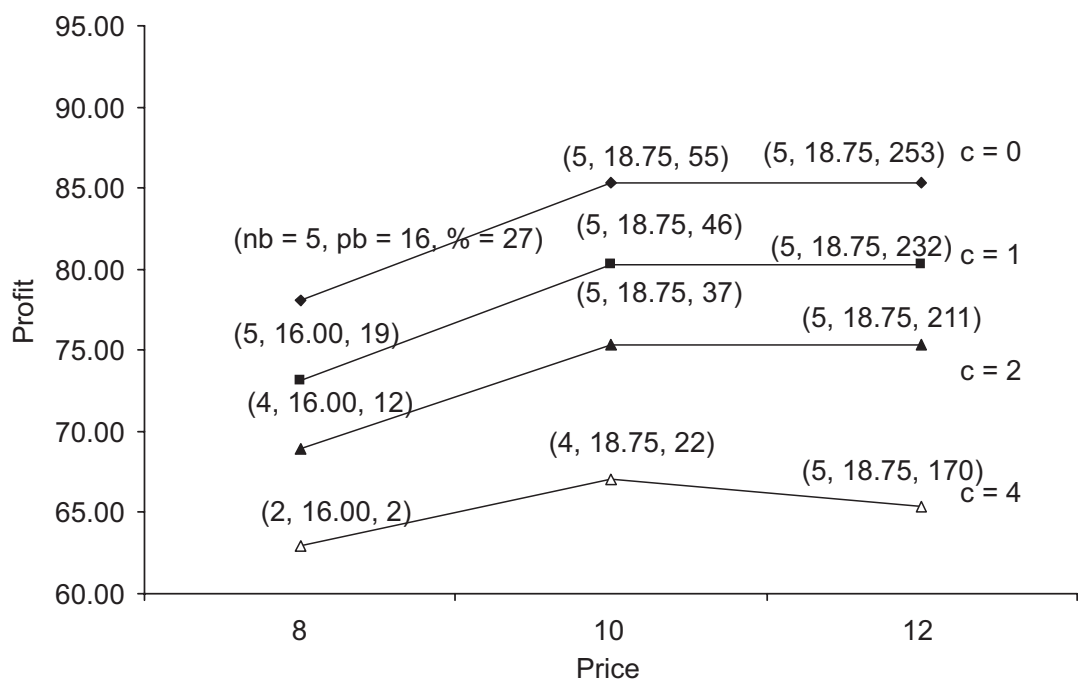

Fig. 4. Profit vs. product price for $\rho=0.9$.

Table 4

The impact of initial inventory levels on the expected profit: $p_{1}=p_{2}=10, \mu_{1}=\mu_{2}=10, \sigma_{1}=\sigma_{2}=2, \theta=0, \lambda=10$.

\begin{tabular}{|c|c|c|c|c|c|c|c|c|c|c|c|}
\hline$Q_{1}=Q_{2}$ & $\rho$ & $c$ & $n_{b}^{*}$ & $p_{b}^{*}$ & $E$ (Profit) & $\%$ & $E\left(x_{1}\right)=E\left(x_{2}\right)$ & $E\left(x_{b}\right)$ & $\ell_{1}=\ell_{2}$ & $\ell_{b}$ & $\ell_{0}$ \\
\hline 5 & -0.9 & 0 & 5 & 19.25 & 93.0645 & 17.74 & 0.00 & 4.52 & 3.34 & 2.82 & 0.50 \\
\hline 5 & -0.9 & 1 & 4 & 19.00 & 88.3828 & 11.82 & 0.76 & 3.07 & 2.97 & 3.68 & 0.38 \\
\hline 5 & -0.9 & 2 & 3 & 19.00 & 84.8695 & 7.37 & 1.50 & 2.37 & 2.97 & 3.68 & 0.38 \\
\hline 5 & -0.9 & 4 & 1 & 19.25 & 80.6290 & 2.01 & 3.12 & 0.92 & 3.34 & 2.82 & 0.50 \\
\hline 5 & 0 & 0 & 5 & 18.75 & 87.1901 & 27.27 & 0.00 & 3.55 & 1.33 & 5.13 & 2.21 \\
\hline 5 & 0 & 1 & 4 & 18.75 & 83.0791 & 21.26 & 0.77 & 3.39 & 1.33 & 5.13 & 2.21 \\
\hline 5 & 0 & 2 & 3 & 19.00 & 79.1586 & 15.54 & 1.53 & 2.73 & 1.54 & 4.61 & 2.31 \\
\hline 5 & 0 & 4 & 2 & 19.25 & 73.3638 & 7.08 & 2.21 & 1.89 & 1.77 & 4.07 & 2.39 \\
\hline 5 & 0.9 & 0 & 5 & 18.75 & 85.3171 & 55.23 & 0.00 & 4.54 & 0.06 & 6.19 & 3.68 \\
\hline 5 & 0.9 & 1 & 5 & 18.75 & 80.3171 & 46.13 & 0.00 & 4.54 & 0.06 & 6.19 & 3.68 \\
\hline 5 & 0.9 & 2 & 5 & 18.75 & 75.3171 & 37.03 & 0.00 & 4.54 & 0.06 & 6.19 & 3.68 \\
\hline 5 & 0.9 & 4 & 4 & 18.75 & 67.0220 & 21.94 & 0.59 & 3.79 & 0.06 & 6.19 & 3.68 \\
\hline 10 & -0.9 & 0 & 10 & 18.50 & 157.3871 & 70.09 & 0.00 & 8.34 & 2.23 & 5.36 & 0.17 \\
\hline 10 & -0.9 & 1 & 10 & 18.50 & 147.3871 & 59.28 & 0.00 & 8.34 & 2.23 & 5.36 & 0.17 \\
\hline 10 & -0.9 & 2 & 10 & 18.50 & 137.3871 & 48.48 & 0.00 & 8.34 & 2.23 & 5.36 & 0.17 \\
\hline 10 & -0.9 & 4 & 10 & 18.50 & 117.3871 & 26.86 & 0.00 & 8.34 & 2.23 & 5.36 & 0.17 \\
\hline 10 & 0 & 0 & 10 & 16.75 & 135.1856 & 80.44 & 0.00 & 7.95 & 0.26 & 8.38 & 1.10 \\
\hline 10 & 0 & 1 & 10 & 16.75 & 125.1856 & 67.09 & 0.00 & 7.95 & 0.26 & 8.38 & 1.10 \\
\hline 10 & 0 & 2 & 9 & 16.75 & 116.1115 & 54.98 & 0.40 & 7.52 & 0.26 & 8.38 & 1.10 \\
\hline 10 & 0 & 4 & 8 & 17.00 & 99.7217 & 33.10 & 0.70 & 6.93 & 0.33 & 8.10 & 1.24 \\
\hline 10 & 0.9 & 0 & 10 & 16.50 & 126.7549 & 121.77 & 0.00 & 7.68 & 0.00 & 8.15 & 1.85 \\
\hline 10 & 0.9 & 1 & 10 & 16.50 & 116.7549 & 104.27 & 0.00 & 7.68 & 0.00 & 8.15 & 1.85 \\
\hline 10 & 0.9 & 2 & 9 & 16.50 & 107.3775 & 87.87 & 0.18 & 7.38 & 0.00 & 8.15 & 1.85 \\
\hline 10 & 0.9 & 4 & 8 & 16.50 & 90.1415 & 57.71 & 0.37 & 6.95 & 0.00 & 8.15 & 1.85 \\
\hline
\end{tabular}

which increase as the correlation coefficient decreases. The highest profits and bundle prices are attained when the reservation prices are negatively correlated. This observation that bundling is more beneficial with negatively correlated reservation prices is also made in earlier research and is attributed to the fact that the reservation price distribution of the bundle has the smallest variance in this case (see e.g., Adams and Yellen, 1976; Schmalensee, 1984; Salinger, 1995; Bulut et al., 2009). Another general observation is that the optimal number of bundles and the corresponding percentage increase in the profit compared to the unbundling case increases as the correlation coefficient increases. We observe that more bundles with more reduced bundle prices are desired as the correlation coefficient increases. In general more bundles become optimal as initial stock levels, arrival rates, individual product prices and variance of the bundle reservation price increase. For all levels of different factors, except the variance of the reservation price, lower bundle prices are charged for higher correlation 
Table 5

The impact of variance of reservation price distributions on the expected profit: $p_{1}=p_{2}=10, \mu_{1}=\mu_{2}=10, \theta=0, Q_{1}=Q_{2}=5, \lambda=10$.

\begin{tabular}{|c|c|c|c|c|c|c|c|c|c|c|c|}
\hline$\sigma_{1}=\sigma_{2}$ & $\rho$ & $c$ & $n_{b}^{*}$ & $p_{b}^{*}$ & $E$ (Profit) & $\%$ & $E\left(x_{1}\right)=E\left(x_{2}\right)$ & $E\left(x_{b}\right)$ & $\ell_{1}=\ell_{2}$ & $\ell_{b}$ & $\ell_{0}$ \\
\hline 1 & -0.9 & 0 & 5 & 19.50 & 95.3845 & 20.68 & 0.00 & 4.14 & 2.97 & 3.68 & 0.38 \\
\hline 1 & -0.9 & 1 & 5 & 19.50 & 90.3845 & 14.35 & 0.00 & 4.14 & 2.97 & 3.68 & 0.38 \\
\hline 1 & -0.9 & 2 & 3 & 19.25 & 86.3451 & 9.24 & 1.56 & 2.59 & 2.23 & 5.36 & 0.17 \\
\hline 1 & -0.9 & 4 & 2 & 19.50 & 81.3399 & 2.91 & 2.34 & 1.75 & 2.97 & 3.68 & 0.38 \\
\hline 1 & 0 & 0 & 5 & 19.00 & 91.0916 & 32.96 & 0.00 & 3.83 & 0.79 & 6.59 & 1.82 \\
\hline 1 & 0 & 1 & 4 & 19.00 & 86.1757 & 25.78 & 0.81 & 3.76 & 0.79 & 6.59 & 1.82 \\
\hline 1 & 0 & 2 & 4 & 19.00 & 82.1757 & 19.95 & 0.81 & 3.76 & 0.79 & 6.59 & 1.82 \\
\hline 1 & 0 & 4 & 3 & 19.00 & 75.1321 & 9.66 & 1.55 & 2.94 & 0.79 & 6.59 & 1.82 \\
\hline 1 & 0.9 & 0 & 5 & 18.75 & 89.4106 & 62.68 & 0.00 & 4.77 & 0.00 & 7.39 & 2.61 \\
\hline 1 & 0.9 & 1 & 5 & 18.75 & 84.4106 & 53.58 & 0.00 & 4.77 & 0.00 & 7.39 & 2.61 \\
\hline 1 & 0.9 & 2 & 5 & 18.75 & 79.4106 & 44.48 & 0.00 & 4.77 & 0.00 & 7.39 & 2.61 \\
\hline 1 & 0.9 & 4 & 4 & 18.75 & 70.6432 & 28.53 & 0.67 & 3.91 & 0.00 & 7.39 & 2.61 \\
\hline 2 & -0.9 & 0 & 5 & 19.25 & 93.0645 & 17.74 & 0.00 & 4.52 & 3.34 & 2.82 & 0.50 \\
\hline 2 & -0.9 & 1 & 4 & 19.00 & 88.3828 & 11.82 & 0.76 & 3.07 & 2.97 & 3.68 & 0.38 \\
\hline 2 & -0.9 & 2 & 3 & 19.00 & 84.8695 & 7.37 & 1.50 & 2.37 & 2.97 & 3.68 & 0.38 \\
\hline 2 & -0.9 & 4 & 1 & 19.25 & 80.6290 & 2.01 & 3.12 & 0.92 & 3.34 & 2.82 & 0.50 \\
\hline 2 & 0 & 0 & 5 & 18.75 & 87.1901 & 27.27 & 0.00 & 3.55 & 1.33 & 5.13 & 2.21 \\
\hline 2 & 0 & 1 & 4 & 18.75 & 83.0791 & 21.26 & 0.77 & 3.39 & 1.33 & 5.13 & 2.21 \\
\hline 2 & 0 & 2 & 3 & 19.00 & 79.1586 & 15.54 & 1.53 & 2.73 & 1.54 & 4.61 & 2.31 \\
\hline 2 & 0 & 4 & 2 & 19.25 & 73.3638 & 7.08 & 2.21 & 1.89 & 1.77 & 4.07 & 2.39 \\
\hline 2 & 0.9 & 0 & 5 & 18.75 & 85.3171 & 55.23 & 0.00 & 4.54 & 0.06 & 6.19 & 3.68 \\
\hline 2 & 0.9 & 1 & 5 & 18.75 & 80.3171 & 46.13 & 0.00 & 4.54 & 0.06 & 6.19 & 3.68 \\
\hline 2 & 0.9 & 2 & 5 & 18.75 & 75.3171 & 37.03 & 0.00 & 4.54 & 0.06 & 6.19 & 3.68 \\
\hline 2 & 0.9 & 4 & 4 & 18.75 & 67.0220 & 21.94 & 0.59 & 3.79 & 0.06 & 6.19 & 3.68 \\
\hline 3 & -0.9 & 0 & 5 & 19.00 & 91.2895 & 15.50 & 0.00 & 4.59 & 3.46 & 2.54 & 0.54 \\
\hline 3 & -0.9 & 1 & 4 & 19.00 & 86.8313 & 9.86 & 0.89 & 3.42 & 3.46 & 2.54 & 0.54 \\
\hline 3 & -0.9 & 2 & 3 & 18.75 & 83.6536 & 5.83 & 1.54 & 2.38 & 3.22 & 3.10 & 0.46 \\
\hline 3 & -0.9 & 4 & 1 & 18.50 & 80.0610 & 1.29 & 3.18 & 0.95 & 2.97 & 3.68 & 0.38 \\
\hline 3 & 0 & 0 & 4 & 18.75 & 85.1352 & 24.27 & 0.77 & 3.18 & 1.69 & 4.25 & 2.37 \\
\hline 3 & 0 & 1 & 4 & 18.75 & 81.1352 & 18.43 & 0.77 & 3.18 & 1.69 & 4.25 & 2.37 \\
\hline 3 & 0 & 2 & 3 & 19.00 & 77.9490 & 13.78 & 1.52 & 2.59 & 1.85 & 3.89 & 2.41 \\
\hline 3 & 0 & 4 & 2 & 19.50 & 72.9087 & 6.42 & 2.21 & 1.79 & 2.17 & 3.18 & 2.48 \\
\hline 3 & 0.9 & 0 & 5 & 19.00 & 83.2592 & 51.48 & 0.00 & 4.28 & 0.24 & 5.44 & 4.09 \\
\hline 3 & 0.9 & 1 & 5 & 19.00 & 78.2592 & 42.39 & 0.00 & 4.28 & 0.24 & 5.44 & 4.09 \\
\hline 3 & 0.9 & 2 & 4 & 19.50 & 73.6754 & 34.05 & 0.61 & 3.55 & 0.43 & 4.90 & 4.23 \\
\hline 3 & 0.9 & 4 & 3 & 20.00 & 65.8112 & 19.74 & 1.17 & 2.72 & 0.72 & 4.28 & 4.28 \\
\hline
\end{tabular}

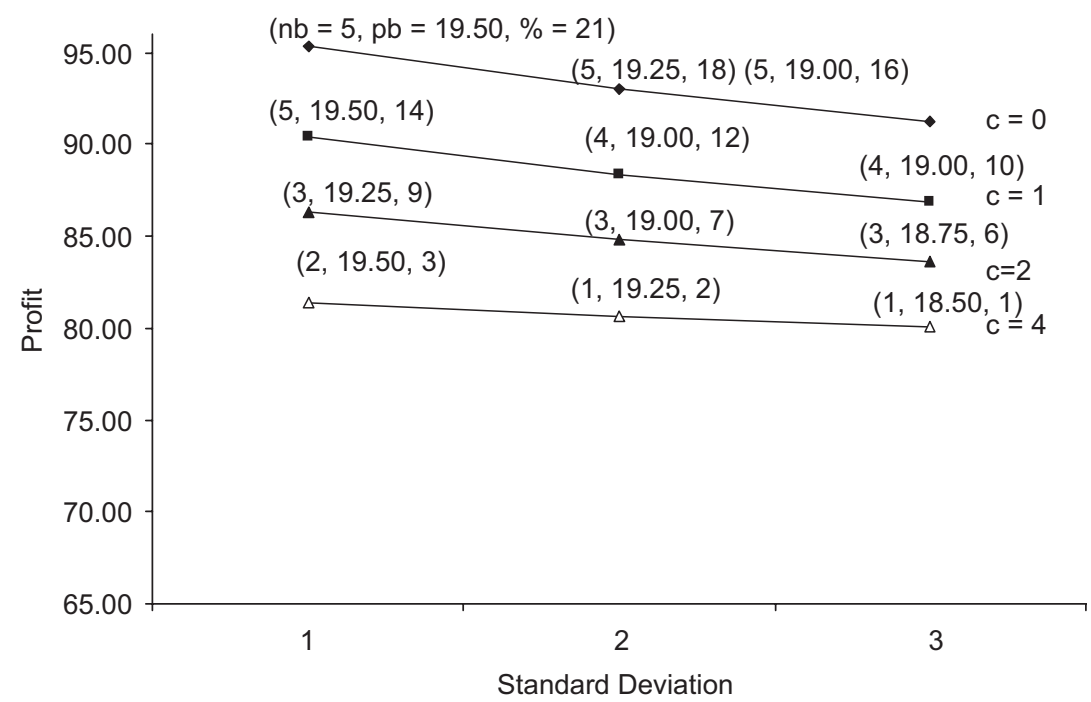

Fig. 5. Profit vs. standard deviation for $\rho=-0.9$.

coefficient, whereas when the standard deviation of the reservation price is high, the bundle price is also high with positive correlation. All these results imply that the variance of the bundle reservation price has a crucial, non-trivial impact on the implementation of bundling policies. 


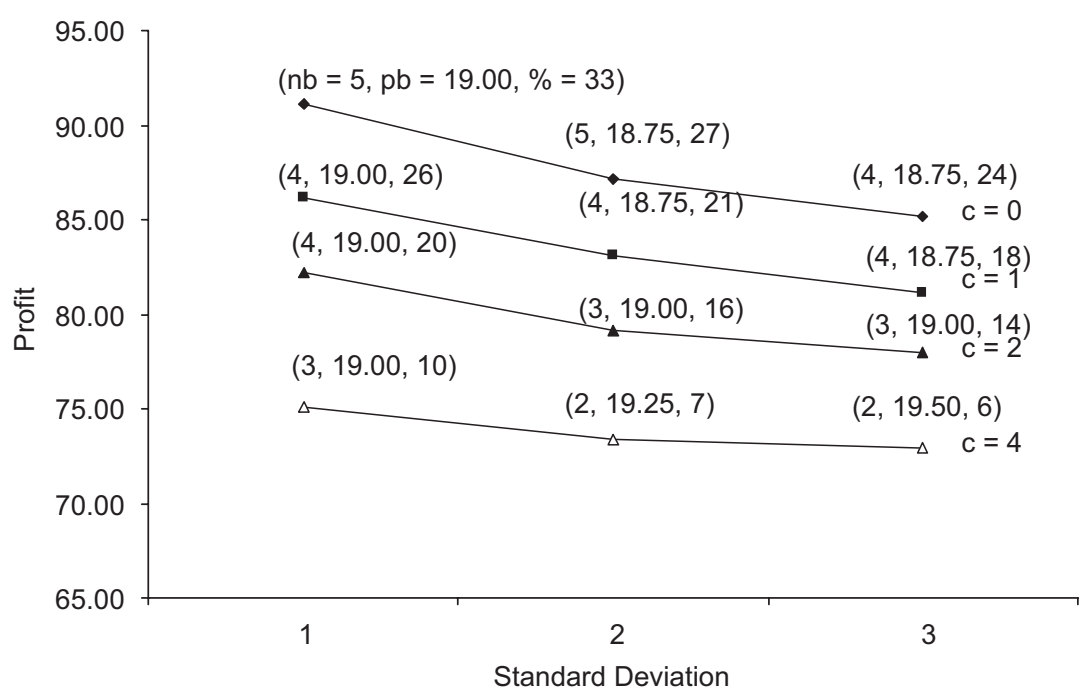

Fig. 6. Profit vs. standard deviation for $\rho=0$.

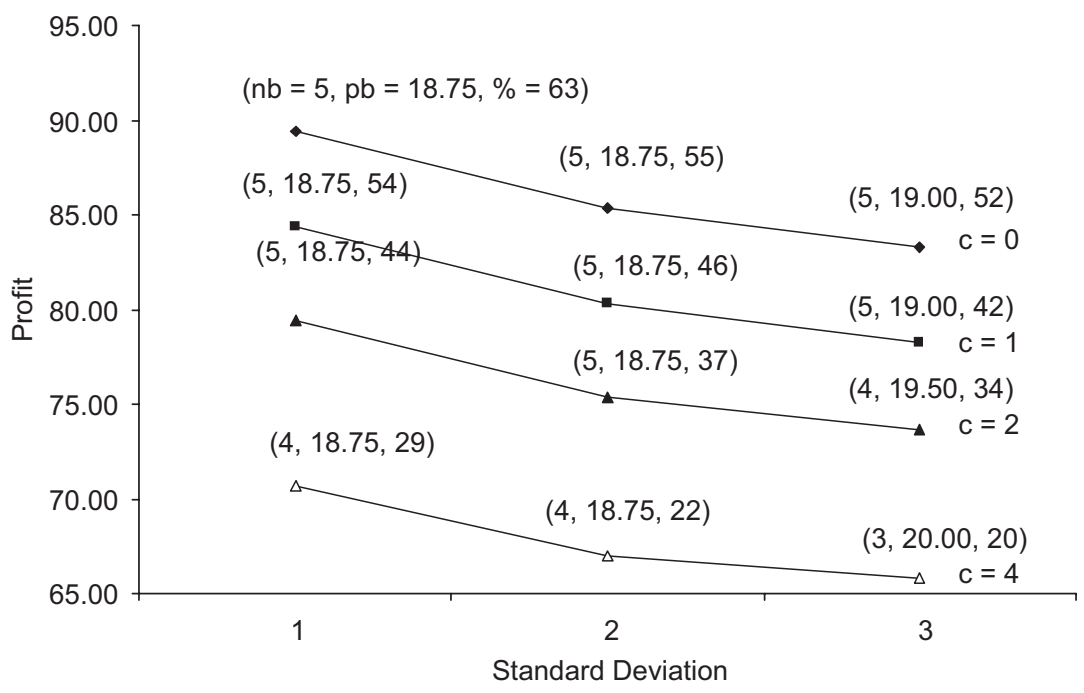

Fig. 7. Profit vs. standard deviation for $\rho=0.9$.

\subsection{Substitutability and complementarity}

We now investigate the effects of product substitutability and complementarity. The degree of contingency, $\theta$ considered in this section has five different values: -0.1 , $-0.05,0,0.05$ and 0.1 . The other parameters are same as the parameters in the base case. The results for the correlation coefficient, $\rho$, of $-0.9,0$ and 0.9 are tabulated in Tables 7-9, respectively. Figs. 11-13 depict the same results graphically.

Expected profit is an increasing function of degree of contingency under all correlation coefficient values. For the positive degree of contingency case (superadditive reservation prices), customer willingness to purchase the bundle is higher than the negative degree of contingency case (subadditive reservation prices). Therefore for all correlation values and bundle formation costs, we see that the retailer is forming more bundles, or charging higher prices for the bundle or both as the degree of contingency increases. The highest jump in the profits occur when the degree of contingency increases to 0 from -0.05 which shows that the product substitutability has a significant impact on the efficiency of bundling and pricing. The impact of the degree of contingency is more pronounced when the product prices are uncorrelated or positively correlated. It seems that the retailer is already able to generate high profits through bundling when the product reservation prices are negatively correlated and the impact of product complementarity is not that significant. 
Table 6

The impact of arrival rate on the expected profit: $p_{1}=p_{2}=10, \mu_{1}=\mu_{2}=10, \sigma_{1}=\sigma_{2}=2, \theta=0, Q_{1}=Q_{2}=5$.

\begin{tabular}{|c|c|c|c|c|c|c|c|c|c|c|c|}
\hline$\lambda$ & $\rho$ & c & $n_{b}^{*}$ & $p_{b}^{*}$ & $E$ (Profit) & $\%$ & $E\left(x_{1}\right)=E\left(x_{2}\right)$ & $E\left(x_{b}\right)$ & $\ell_{1}=\ell_{2}$ & $\ell_{b}$ & $\ell_{0}$ \\
\hline 5 & -0.9 & 0 & 5 & 18.50 & 74.2297 & 63.10 & 0.00 & 3.89 & 1.12 & 2.68 & 0.09 \\
\hline 5 & -0.9 & 1 & 5 & 18.50 & 69.2297 & 52.11 & 0.00 & 3.89 & 1.12 & 2.68 & 0.09 \\
\hline 5 & -0.9 & 2 & 5 & 18.50 & 64.2297 & 41.13 & 0.00 & 3.89 & 1.12 & 2.68 & 0.09 \\
\hline 5 & -0.9 & 4 & 5 & 18.50 & 54.2297 & 19.15 & 0.00 & 3.89 & 1.12 & 2.68 & 0.09 \\
\hline 5 & 0 & 0 & 5 & 17.00 & 63.8536 & 71.84 & 0.00 & 3.73 & 0.17 & 4.05 & 0.62 \\
\hline 5 & 0 & 1 & 5 & 17.00 & 58.8536 & 58.38 & 0.00 & 3.73 & 0.17 & 4.05 & 0.62 \\
\hline 5 & 0 & 2 & 4 & 17.00 & 54.2706 & 46.05 & 0.35 & 3.25 & 0.17 & 4.05 & 0.62 \\
\hline 5 & 0 & 4 & 3 & 17.25 & 46.5785 & 25.35 & 0.67 & 2.62 & 0.21 & 3.89 & 0.69 \\
\hline 5 & 0.9 & 0 & 5 & 16.50 & 60.0115 & 110.60 & 0.00 & 3.64 & 0.00 & 4.08 & 0.92 \\
\hline 5 & 0.9 & 1 & 5 & 16.50 & 55.0115 & 93.06 & 0.00 & 3.64 & 0.00 & 4.08 & 0.92 \\
\hline 5 & 0.9 & 2 & 5 & 16.50 & 50.0115 & 75.51 & 0.00 & 3.64 & 0.00 & 4.08 & 0.92 \\
\hline 5 & 0.9 & 4 & 4 & 16.75 & 41.9065 & 47.07 & 0.20 & 3.21 & 0.00 & 3.99 & 1.01 \\
\hline 10 & -0.9 & 0 & 5 & 19.25 & 93.0645 & 17.74 & 0.00 & 4.52 & 3.34 & 2.82 & 0.50 \\
\hline 10 & -0.9 & 1 & 4 & 19.00 & 88.3828 & 11.82 & 0.76 & 3.07 & 2.97 & 3.68 & 0.38 \\
\hline 10 & -0.9 & 2 & 3 & 19.00 & 84.8695 & 7.37 & 1.50 & 2.37 & 2.97 & 3.68 & 0.38 \\
\hline 10 & -0.9 & 4 & 1 & 19.25 & 80.6290 & 2.01 & 3.12 & 0.92 & 3.34 & 2.82 & 0.50 \\
\hline 10 & 0 & 0 & 5 & 18.75 & 87.1901 & 27.27 & 0.00 & 3.55 & 1.33 & 5.13 & 2.21 \\
\hline 10 & 0 & 1 & 4 & 18.75 & 83.0791 & 21.26 & 0.77 & 3.39 & 1.33 & 5.13 & 2.21 \\
\hline 10 & 0 & 2 & 3 & 19.00 & 79.1586 & 15.54 & 1.53 & 2.73 & 1.54 & 4.61 & 2.31 \\
\hline 10 & 0 & 4 & 2 & 19.25 & 73.3638 & 7.08 & 2.21 & 1.89 & 1.77 & 4.07 & 2.39 \\
\hline 10 & 0.9 & 0 & 5 & 18.75 & 85.3171 & 55.23 & 0.00 & 4.54 & 0.06 & 6.19 & 3.68 \\
\hline 10 & 0.9 & 1 & 5 & 18.75 & 80.3171 & 46.13 & 0.00 & 4.54 & 0.06 & 6.19 & 3.68 \\
\hline 10 & 0.9 & 2 & 5 & 18.75 & 75.3171 & 37.03 & 0.00 & 4.54 & 0.06 & 6.19 & 3.68 \\
\hline 10 & 0.9 & 4 & 4 & 18.75 & 67.0220 & 21.94 & 0.59 & 3.79 & 0.06 & 6.19 & 3.68 \\
\hline 15 & -0.9 & 0 & 3 & 19.75 & 97.3646 & 3.60 & 1.75 & 2.58 & 6.01 & 1.94 & 1.03 \\
\hline 15 & -0.9 & 1 & 1 & 19.75 & 95.2020 & 1.29 & 2.66 & 0.68 & 6.01 & 1.94 & 1.03 \\
\hline 15 & -0.9 & 2 & 1 & 19.75 & 94.2020 & 0.23 & 2.66 & 0.68 & 6.01 & 1.94 & 1.03 \\
\hline 15 & -0.9 & 4 & 0 & - & 93.9854 & 0.00 & 4.70 & 0.00 & 6.96 & 0.00 & 1.08 \\
\hline 15 & 0 & 0 & 4 & 19.75 & 95.9375 & 9.93 & 0.64 & 2.50 & 3.38 & 4.51 & 3.73 \\
\hline 15 & 0 & 1 & 2 & 20.00 & 92.8984 & 6.44 & 2.23 & 1.55 & 3.75 & 3.75 & 3.75 \\
\hline 15 & 0 & 2 & 2 & 20.00 & 90.8984 & 4.15 & 2.23 & 1.55 & 3.75 & 3.75 & 3.75 \\
\hline 15 & 0 & 4 & 1 & 20.00 & 88.6177 & 1.54 & 3.28 & 0.89 & 3.75 & 3.75 & 3.75 \\
\hline 15 & 0.9 & 0 & 5 & 20.00 & 95.6171 & 27.08 & 0.00 & 3.43 & 1.08 & 6.42 & 6.42 \\
\hline 15 & 0.9 & 1 & 4 & 20.00 & 90.9569 & 20.89 & 0.82 & 3.60 & 1.08 & 6.42 & 6.42 \\
\hline 15 & 0.9 & 2 & 4 & 20.00 & 86.9569 & 15.57 & 0.82 & 3.60 & 1.08 & 6.42 & 6.42 \\
\hline 15 & 0.9 & 4 & 3 & 20.00 & 80.2742 & 6.69 & 1.65 & 2.92 & 1.08 & 6.42 & 6.42 \\
\hline
\end{tabular}

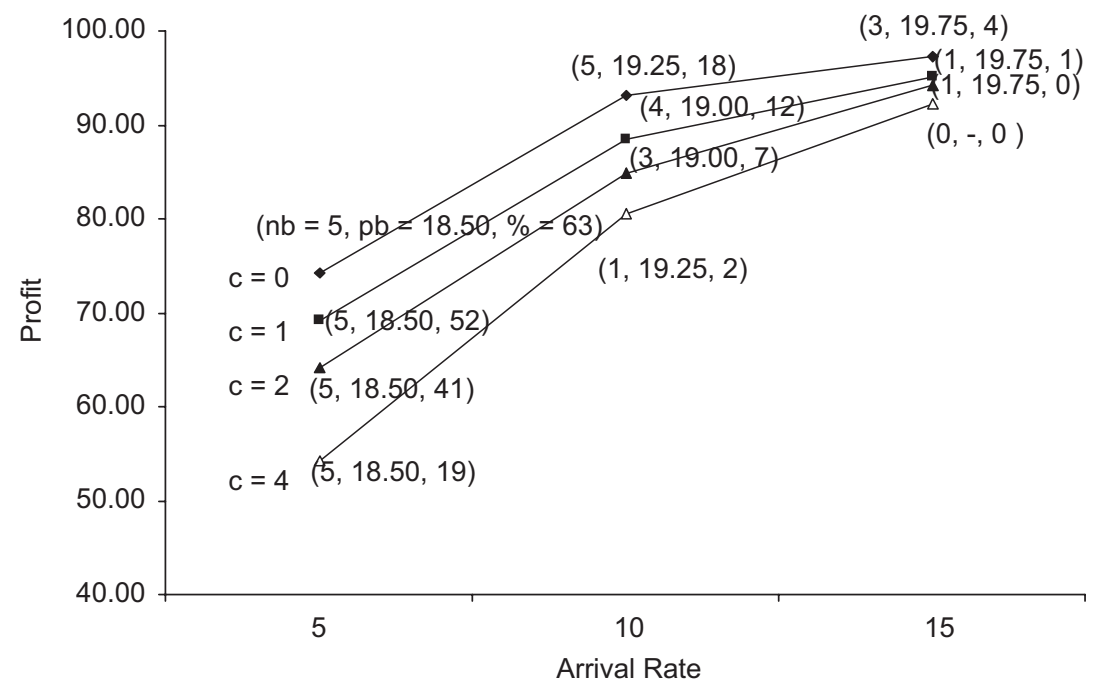

Fig. 8. Profit vs. arrival rate for $\rho=-0.9$.

\section{Conclusion}

We consider a retail firm that sells two types of perishable products in a single period not only as independent items but also as a bundle. Our emphasis is on understanding the bundling practices on the inventory and pricing decisions of the firm. We study the bundle formation and pricing problem of two products facing 


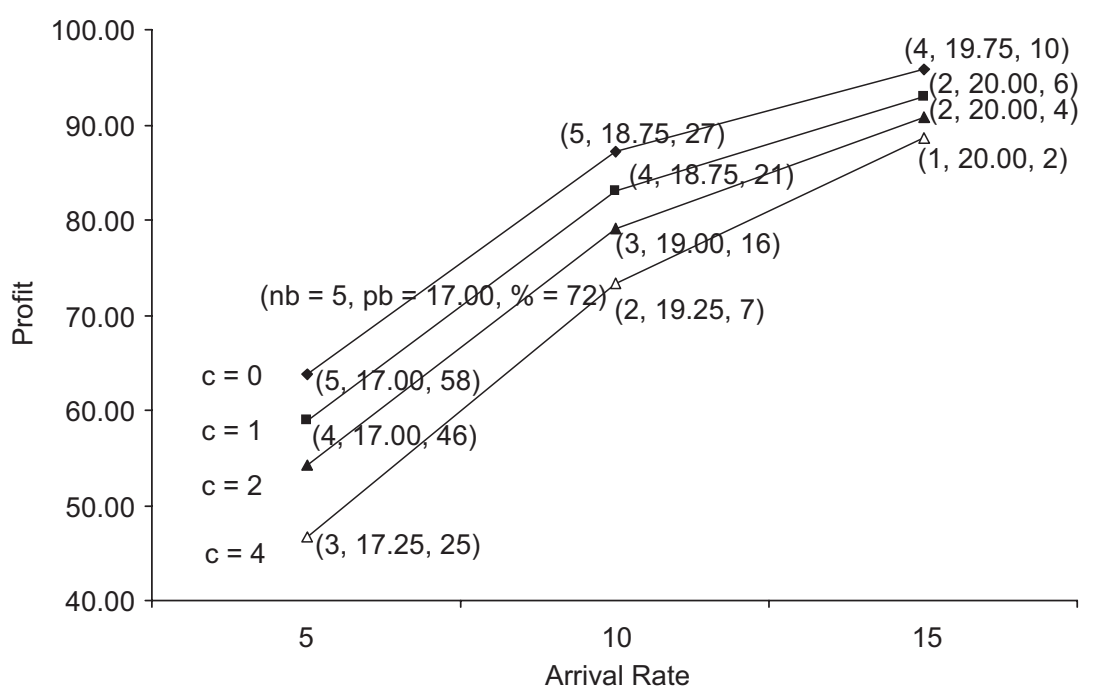

Fig. 9. Profit vs. arrival rate for $\rho=0$.

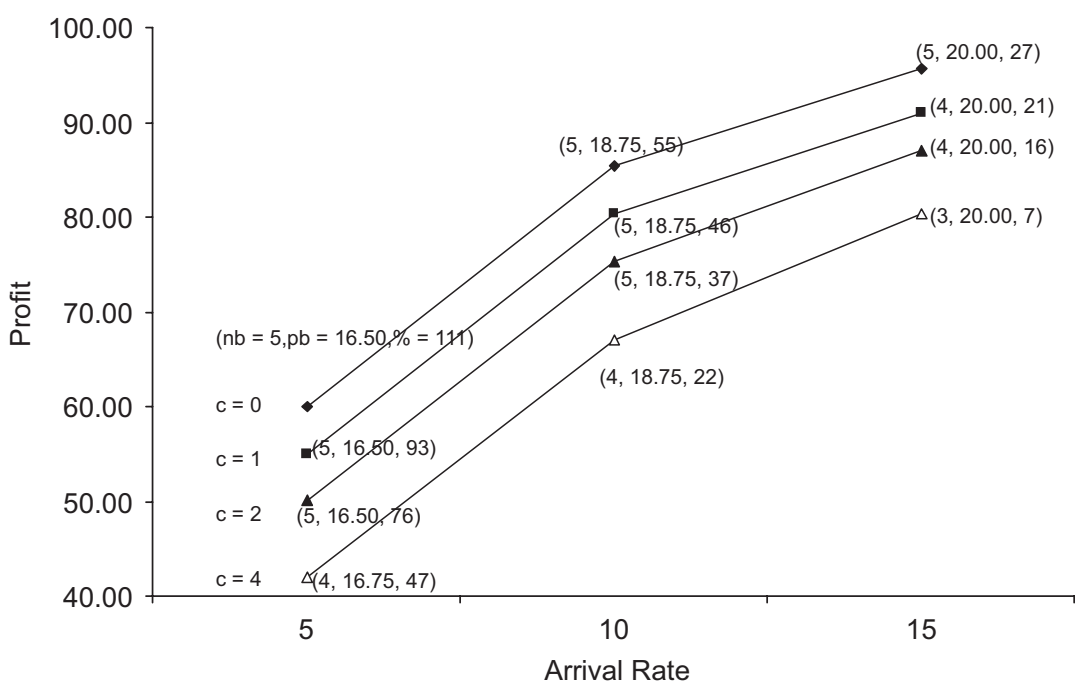

Fig. 10. Profit vs. arrival rate for $\rho=0.9$.

random demand, under inventory constraints over a finite selling horizon. After the retailer decides the number of bundles to be formed at the beginning of the season, no new bundles are formed and none of the bundles are unbundled to offer individual products during the season. Bundle formation costs are also included in the model.

Our numerical study shows that the optimal bundle price and expected profits are decreasing functions of the correlation coefficient. While the bundle formation cost has a significant impact on total profit, the impact on the number of bundles depends on the correlation coefficient. With negative correlation, bundling cost has a significant impact on the number of bundles. However with positive correlation, this effect is negligible. When the individual product prices are set below the mean reservation price, the retailer sets the highest possible bundle price and offers individual products as well as the bundle (mixed bundling). When the individual product prices are high, the retailer sets a bundle price such that only bundles are sold (pure bundling).

Other findings include the fact that the expected profit and the optimal number of bundles formed decreases as the variance of the reservation price distribution increases. The impact of the variance of the reservation price distribution on the expected profit is much 
Table 7

The impact of degree of contingency on the expected profit for $\rho=-0.9: p_{1}=p_{2}=10, \mu_{1}=\mu_{2}=10, \sigma_{1}=\sigma_{2}=2, Q_{1}=Q_{2}=5, \lambda=10$.

\begin{tabular}{|c|c|c|c|c|c|c|c|c|c|c|}
\hline$c$ & $\theta$ & $n_{b}^{*}$ & $p_{b}^{*}$ & $E$ (Profit) & $\%$ & $E\left(x_{1}\right)=E\left(x_{2}\right)$ & $E\left(x_{b}\right)$ & $\ell_{1}=\ell_{2}$ & $\ell_{b}$ & $\ell_{0}$ \\
\hline 0 & -0.10 & 5 & 17.50 & 80.4776 & 1.82 & 0.00 & 4.53 & 3.74 & 1.93 & 0.59 \\
\hline 0 & -0.05 & 5 & 18.50 & 84.7307 & 7.20 & 0.00 & 4.51 & 3.72 & 1.97 & 0.60 \\
\hline 0 & 0.00 & 5 & 19.25 & 93.0645 & 17.74 & 0.00 & 4.52 & 3.34 & 2.82 & 0.50 \\
\hline 0 & 0.05 & 5 & 20.00 & 97.6682 & 23.57 & 0.00 & 4.13 & 2.96 & 3.68 & 0.40 \\
\hline 0 & 0.10 & 5 & 20.00 & 98.9330 & 25.17 & 0.00 & 2.84 & 1.60 & 6.71 & 0.09 \\
\hline 1 & -0.10 & 0 & - & 79.0416 & 0.00 & 3.95 & 0.00 & 4.64 & 0.00 & 0.72 \\
\hline 1 & -0.05 & 2 & 18.00 & 79.9001 & 1.09 & 2.33 & 1.59 & 2.98 & 3.69 & 0.35 \\
\hline 1 & 0.00 & 4 & 19.00 & 88.3828 & 11.82 & 0.76 & 3.07 & 2.97 & 3.68 & 0.38 \\
\hline 1 & 0.05 & 5 & 20.00 & 92.6682 & 17.24 & 0.00 & 4.13 & 2.96 & 3.68 & 0.40 \\
\hline 1 & 0.10 & 5 & 20.00 & 93.9330 & 18.84 & 0.00 & 2.84 & 1.60 & 6.71 & 0.09 \\
\hline 2 & -0.10 & 0 & - & 79.0416 & 0.00 & 3.95 & 0.00 & 4.64 & 0.00 & 0.72 \\
\hline 2 & -0.05 & 0 & - & 79.0416 & 0.00 & 3.95 & 0.00 & 4.64 & 0.00 & 0.72 \\
\hline 2 & 0.00 & 3 & 19.00 & 84.8695 & 7.37 & 1.50 & 2.37 & 2.97 & 3.68 & 0.38 \\
\hline 2 & 0.05 & 5 & 20.00 & 87.6682 & 10.91 & 0.00 & 4.13 & 2.96 & 3.68 & 0.40 \\
\hline 2 & 0.10 & 4 & 20.00 & 89.3489 & 13.04 & 0.75 & 3.22 & 1.60 & 6.71 & 0.09 \\
\hline 4 & -0.10 & 0 & - & 79.0416 & 0.00 & 3.95 & 0.00 & 4.64 & 0.00 & 0.72 \\
\hline 4 & -0.05 & 0 & - & 79.0416 & 0.00 & 3.95 & 0.00 & 4.64 & 0.00 & 0.72 \\
\hline 4 & 0.00 & 1 & 19.25 & 80.6290 & 2.01 & 3.12 & 0.92 & 3.34 & 2.82 & 0.50 \\
\hline 4 & 0.05 & 2 & 20.00 & 82.2348 & 4.04 & 2.34 & 1.76 & 2.96 & 3.68 & 0.40 \\
\hline 4 & 0.10 & 2 & 20.00 & 82.7798 & 4.73 & 2.53 & 1.98 & 1.60 & 6.71 & 0.09 \\
\hline
\end{tabular}

Table 8

The impact of degree of contingency on the expected profit for $\rho=0: p_{1}=p_{2}=10, \mu_{1}=\mu_{2}=10, \sigma_{1}=\sigma_{2}=2, Q_{1}=Q_{2}=5, \lambda=10$.

\begin{tabular}{|c|c|c|c|c|c|c|c|c|c|c|}
\hline$c$ & $\theta$ & $n_{b}^{*}$ & $p_{b}^{*}$ & $E$ (Profit) & $\%$ & $E\left(x_{1}\right)=E\left(x_{2}\right)$ & $E\left(x_{b}\right)$ & $\ell_{1}=\ell_{2}$ & $\ell_{b}$ & $\ell_{0}$ \\
\hline 0 & -0.10 & 3 & 16.75 & 71.6768 & 4.62 & 1.54 & 2.55 & 1.36 & 5.13 & 2.15 \\
\hline 0 & -0.05 & 3 & 17.50 & 74.2086 & 8.32 & 1.55 & 2.71 & 1.14 & 5.67 & 2.05 \\
\hline 0 & 0.00 & 5 & 18.75 & 87.1901 & 27.27 & 0.00 & 3.55 & 1.33 & 5.13 & 2.21 \\
\hline 0 & 0.05 & 5 & 19.75 & 91.5326 & 33.60 & 0.00 & 3.54 & 1.32 & 5.13 & 2.23 \\
\hline 0 & 0.10 & 5 & 20.00 & 95.3458 & 39.17 & 0.00 & 3.80 & 0.82 & 6.44 & 1.92 \\
\hline 1 & -0.10 & 2 & 17.50 & 69.0528 & 0.79 & 2.21 & 1.61 & 2.13 & 3.31 & 2.44 \\
\hline 1 & -0.05 & 3 & 17.50 & 71.2086 & 3.94 & 1.55 & 2.71 & 1.14 & 5.67 & 2.05 \\
\hline 1 & 0.00 & 4 & 18.75 & 83.0791 & 21.26 & 0.77 & 3.39 & 1.33 & 5.13 & 2.21 \\
\hline 1 & 0.05 & 4 & 19.75 & 86.6642 & 26.50 & 0.77 & 3.39 & 1.32 & 5.13 & 2.23 \\
\hline 1 & 0.10 & 5 & 20.00 & 90.3458 & 31.87 & 0.00 & 3.80 & 0.82 & 6.44 & 1.92 \\
\hline 2 & -0.10 & 0 & - & 68.5106 & 0.00 & 3.43 & 0.00 & 3.75 & 0.00 & 2.50 \\
\hline 2 & -0.05 & 2 & 18.50 & 68.7396 & 0.33 & 2.21 & 1.65 & 2.06 & 3.43 & 2.45 \\
\hline 2 & 0.00 & 3 & 19.00 & 79.1586 & 15.54 & 1.53 & 2.73 & 1.54 & 4.61 & 2.31 \\
\hline 2 & 0.05 & 4 & 19.75 & 82.6642 & 20.66 & 0.77 & 3.39 & 1.32 & 5.13 & 2.23 \\
\hline 2 & 0.10 & 4 & 20.00 & 85.7052 & 25.10 & 0.80 & 3.73 & 0.82 & 6.44 & 1.92 \\
\hline 4 & -0.10 & 0 & - & 68.5106 & 0.00 & 3.43 & 0.00 & 3.75 & 0.00 & 2.50 \\
\hline 4 & -0.05 & 0 & - & 68.5106 & 0.00 & 3.43 & 0.00 & 3.75 & 0.00 & 2.50 \\
\hline 4 & 0.00 & 2 & 19.25 & 73.3638 & 7.08 & 2.21 & 1.89 & 1.77 & 4.07 & 2.39 \\
\hline 4 & 0.05 & 3 & 20.00 & 75.9234 & 10.82 & 1.53 & 2.74 & 1.52 & 4.64 & 2.33 \\
\hline 4 & 0.10 & 3 & 20.00 & 77.8449 & 13.62 & 1.55 & 2.94 & 0.82 & 6.44 & 1.92 \\
\hline
\end{tabular}

more significant when there is a positive correlation between the reservation prices. We also perform analysis to investigate the product substitutability and complementarity. For the positive degree of contingency case (superadditive reservation prices), customer willingness to purchase bundle is higher than the negative degree of contingency case (subadditive reservation prices). Therefore for all correlation values and bundle formation costs, we see that the retailer forms more bundles, or charges higher prices for the bundle, or both as the degree of contingency increases. As a result, expected profit is an increasing function of degree of contingency.

As an important future research direction, the single period model in this paper can be extended to a multi-period model, allowing new bundle formations (and perhaps unbundling) and re-pricing at the beginning of each period. Also we can also extend the model to allow for replenishments of individual products at a certain cost. Another important but a complex extension of our work could be the modeling of competition. 
Table 9

The impact of degree of contingency on the expected profit for $\rho=0.9: p_{1}=p_{2}=10, \mu_{1}=\mu_{2}=10, \sigma_{1}=\sigma_{2}=2, Q_{1}=Q_{2}=5, \lambda=10$.

\begin{tabular}{|c|c|c|c|c|c|c|c|c|c|c|}
\hline c & $\theta$ & $n_{b}^{*}$ & $p_{b}^{*}$ & $E$ (Profit) & $\%$ & $E\left(x_{1}\right)=E\left(x_{2}\right)$ & $E\left(x_{b}\right)$ & $\ell_{1}=\ell_{2}$ & $\ell_{b}$ & $\ell_{0}$ \\
\hline 0 & -0.10 & 5 & 15.25 & 73.5533 & 33.82 & 0.00 & 4.82 & 0.00 & 7.83 & 2.17 \\
\hline 0 & -0.05 & 5 & 16.00 & 77.2984 & 40.64 & 0.00 & 4.83 & 0.00 & 7.91 & 2.09 \\
\hline 0 & 0.00 & 5 & 18.75 & 85.3171 & 55.23 & 0.00 & 4.54 & 0.06 & 6.19 & 3.68 \\
\hline 0 & 0.05 & 5 & 19.75 & 89.5730 & 62.97 & 0.00 & 4.52 & 0.07 & 6.13 & 3.73 \\
\hline 0 & 0.10 & 5 & 20.00 & 93.3858 & 69.91 & 0.00 & 4.67 & 0.01 & 6.78 & 3.19 \\
\hline 1 & -0.10 & 5 & 15.25 & 68.5533 & 24.73 & 0.00 & 4.82 & 0.00 & 7.83 & 2.17 \\
\hline 1 & -0.05 & 5 & 16.00 & 72.2984 & 31.54 & 0.00 & 4.83 & 0.00 & 7.91 & 2.09 \\
\hline 1 & 0.00 & 5 & 18.75 & 80.3171 & 46.13 & 0.00 & 4.54 & 0.06 & 6.19 & 3.68 \\
\hline 1 & 0.05 & 5 & 19.75 & 84.5730 & 53.87 & 0.00 & 4.52 & 0.07 & 6.13 & 3.73 \\
\hline 1 & 0.10 & 5 & 20.00 & 88.3858 & 60.81 & 0.00 & 4.67 & 0.01 & 6.78 & 3.19 \\
\hline 2 & -0.10 & 5 & 15.25 & 63.5533 & 15.63 & 0.00 & 4.82 & 0.00 & 7.83 & 2.17 \\
\hline 2 & -0.05 & 5 & 16.00 & 67.2984 & 22.44 & 0.00 & 4.83 & 0.00 & 7.91 & 2.09 \\
\hline 2 & 0.00 & 5 & 18.75 & 75.3171 & 37.03 & 0.00 & 4.54 & 0.06 & 6.19 & 3.68 \\
\hline 2 & 0.05 & 5 & 19.75 & 79.5730 & 44.78 & 0.00 & 4.52 & 0.07 & 6.13 & 3.73 \\
\hline 2 & 0.10 & 5 & 20.00 & 83.3858 & 51.71 & 0.00 & 4.67 & 0.01 & 6.78 & 3.19 \\
\hline 4 & -0.10 & 0 & - & 54.9623 & 0.00 & 2.75 & 0.00 & 2.86 & 0.00 & 4.28 \\
\hline 4 & -0.05 & 4 & 17.75 & 57.4697 & 4.56 & 0.60 & 3.77 & 0.06 & 6.26 & 3.63 \\
\hline 4 & 0.00 & 4 & 18.75 & 67.0220 & 21.94 & 0.59 & 3.79 & 0.06 & 6.19 & 3.68 \\
\hline 4 & 0.05 & 4 & 19.75 & 70.5879 & 28.43 & 0.59 & 3.79 & 0.07 & 6.13 & 3.73 \\
\hline 4 & 0.10 & 4 & 20.00 & 73.7592 & 34.20 & 0.63 & 3.86 & 0.01 & 6.78 & 3.19 \\
\hline
\end{tabular}

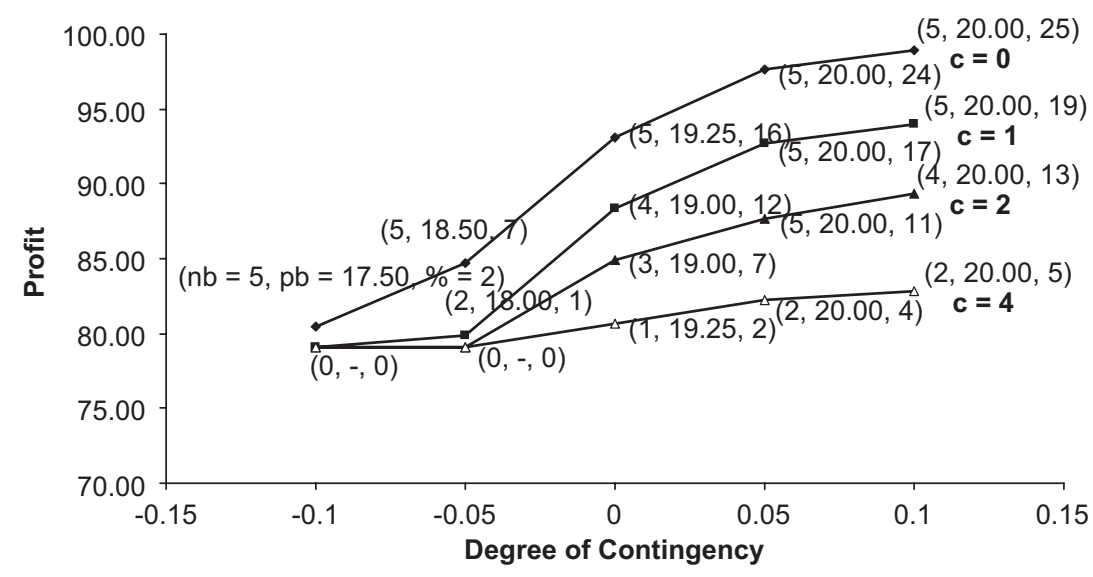

Fig. 11. Profit vs. degree of contingency for $\rho=-0.9$.

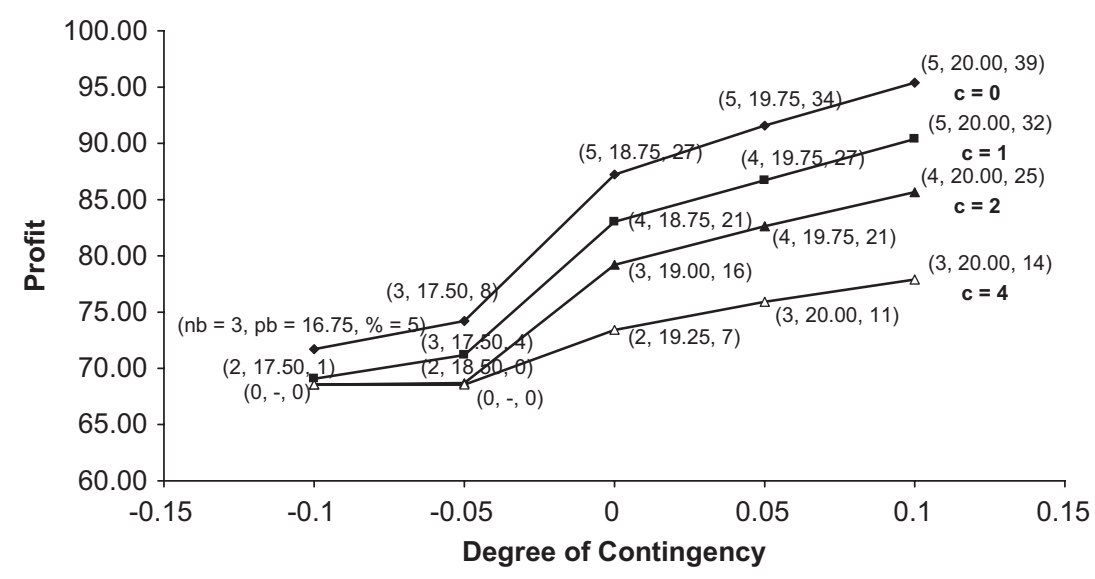

Fig. 12. Profit vs. degree of contingency for $\rho=0$. 


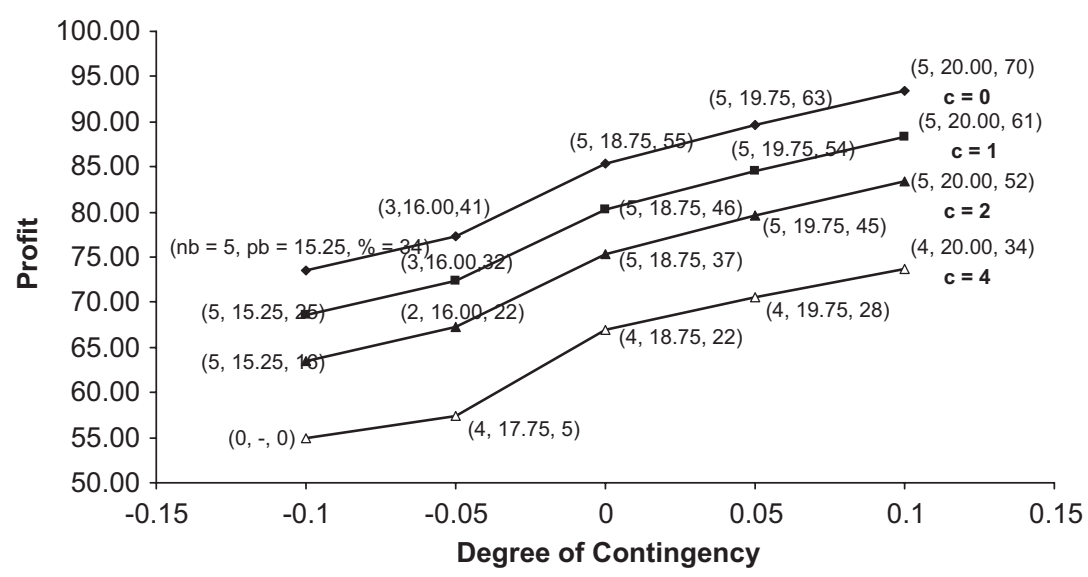

Fig. 13. Profit vs. degree of contingency for $\rho=0.9$.

\section{Appendix A. Purchasing and switching probabilities for} $\theta \neq \mathbf{0}$

\section{A.1. Purchasing probabilities}

Remember that $m_{0}$ denotes the probability of no purchase, $m_{1}, m_{2}, m_{B}$ denote the probability of purchasing Products 1, 2 and the Bundle, respectively. Purchasing probabilities when we have a non-zero degree of contingency can be derived as follows:

$$
\begin{aligned}
m_{0} & =\operatorname{Pr}\left\{R_{1}<p_{1} ; R_{2}<p_{2} ; R_{b}<p_{b}\right\} \\
& =\int_{-\infty}^{p_{1}} \int_{-\infty}^{a_{1}} f_{R_{1}, R_{2}}\left(r_{1}, r_{2}\right) \mathrm{d} r_{1} \mathrm{~d} r_{2} \\
m_{1} & =\operatorname{Pr}\left\{R_{1} \geqslant p_{1} ; R_{1}-p_{1} \geqslant R_{2}-p_{2} ; R_{1}-p_{1} \geqslant R_{b}-p_{b}\right\} \\
& =\int_{p_{1}}^{\infty} \int_{-\infty}^{a_{2}} f_{R_{1}, R_{2}}\left(r_{1}, r_{2}\right) \mathrm{d} r_{1} \mathrm{~d} r_{2} \\
m_{2} & =\operatorname{Pr}\left\{R_{2} \geqslant p_{2} ; R_{2}-p_{2} \geqslant R_{1}-p_{1} ; R_{2}-p_{2} \geqslant R_{b}-p_{b}\right\} \\
& =\int_{p_{2}}^{\infty} \int_{-\infty}^{a_{3}} f_{R_{1}, R_{2}}\left(r_{1}, r_{2}\right) \mathrm{d} r_{2} \mathrm{~d} r_{1} \\
m_{b} & =\operatorname{Pr}\left\{R_{b} \geqslant p_{b} ; R_{b}-p_{b} \geqslant R_{1}-p_{1} ; R_{b}-p_{b} \geqslant R_{2}-p_{2}\right\} \\
& =\int_{p_{b}-p_{2}}^{\infty} \int_{a_{4}}^{\infty} f_{R_{1}, R_{2}}\left(r_{1}, r_{2}\right) \mathrm{d} r_{1} \mathrm{~d} r_{2}
\end{aligned}
$$

where

$$
\begin{aligned}
a_{1}= & \min \left(p_{2},\left(p_{b}-(1+\theta) r_{1}\right) /(1+\theta)\right) \\
a_{2}= & \min \left(r_{1}-p_{1}+p_{2},\left(p_{b}-p_{1}-\theta r_{1}\right) /(1+\theta)\right) \\
a_{3}= & \min \left(r_{2}-p_{2}+p_{1},\left(p_{b}-p_{2}-\theta r_{2}\right) /(1+\theta)\right) \\
a_{4}= & \max \left(\left(p_{b}-(1+\theta) r_{1}\right) /(1+\theta),\left(p_{b}-p_{1}-\theta r_{1}\right) /(1+\theta),\right. \\
& \left.\left(p_{b}-p_{2}-(1+\theta) r_{1}\right) / \theta\right)
\end{aligned}
$$

\section{A.2. Switching probabilities}

One type of product incurs shortage: We first study the case when Product 1 runs out of stock and derive the expression for the probability of switching from Product 1 to the Bundle:

$$
\begin{aligned}
\gamma_{1 B}= & \operatorname{Pr}\left\{R_{b}-p_{b} \geqslant R_{2}-p_{2} ; R_{b} \geqslant p_{b} \mid R_{1}-p_{1} \geqslant R_{b}-p_{b} ;\right. \\
& \left.R_{1}-p_{1} \geqslant R_{2}-p_{2} ; R_{1} \geqslant p_{1}\right\} \\
= & \operatorname{Pr}\left\{\begin{array}{c}
(1+\theta)\left(R_{1}+R_{2}\right)-p_{b} \geqslant R_{2}-p_{2} ; \\
(1+\theta)\left(R_{1}+R_{2}\right) \geqslant p_{b} ; \\
R_{1}-p_{1} \geqslant(1+\theta)\left(R_{1}+R_{2}\right)-p_{b} ; \\
R_{1}-p_{1} \geqslant R_{2}-p_{2} ; R_{1} \geqslant p_{1}
\end{array}\right\} / m_{1} .
\end{aligned}
$$

For $\theta>0$, this expression can be written as

$$
\begin{aligned}
\gamma_{1 B}= & \operatorname{Pr}\left\{R_{2} \geqslant \frac{p_{b}-p_{2}-(1+\theta) R_{1}}{\theta} ; R_{2} \geqslant \frac{p_{b}}{1+\theta}-R_{1} ;\right. \\
& \left.\frac{p_{b}-\theta R_{1}-p_{1}}{1+\theta} \geqslant R_{2} ; R_{1}-p_{1}+p_{2} \geqslant R_{2} ; R_{1} \geqslant p_{1}\right\} / m_{1} \\
= & \operatorname{Pr}\left\{R_{2} \geqslant \max \left(\frac{p_{b}-p_{2}-(1+\theta) R_{1}}{\theta}, \frac{p_{b}}{1+\theta}-R_{1}\right) ;\right. \\
& \left.R_{2} \leqslant \min \left(\frac{p_{b}-\theta R_{1}-p_{1}}{1+\theta}, R_{1}-p_{1}+p_{2}\right) ; R_{1} \geqslant p_{1}\right\} / m_{1} \\
= & \int_{p_{1}}^{\infty} \int_{\max \left(\left(p_{b}-p_{2}-(1+\theta) r_{1}\right) / \theta, p_{b} /(1+\theta)-r_{1}\right)}^{\min \left(\left(p_{b}-\theta r_{1}-p_{1}\right) /(1+\theta), r_{1}-p_{1}+p_{2}\right)} f_{R_{1}, R_{2}}\left(r_{1}, r_{2}\right) \mathrm{d} r_{1} \mathrm{~d} r_{2} / m_{1} .
\end{aligned}
$$

For $\theta<0$, this expression can be written as

$$
\begin{aligned}
\gamma_{1 B}= & \operatorname{Pr}\left\{R_{2} \leqslant \frac{p_{b}-p_{2}-(1+\theta) R_{1}}{\theta} ; R_{2} \geqslant \frac{p_{b}}{1+\theta}-R_{1} ;\right. \\
& \left.\frac{p_{b}-\theta R_{1}-p_{1}}{1+\theta} \geqslant R_{2} ; R_{1}-p_{1}+p_{2} \geqslant R_{2} ; R_{1} \geqslant p_{1}\right\} / m_{1} \\
= & \operatorname{Pr}\left\{R_{2} \geqslant \frac{p_{b}}{(1+\theta)}-R_{1} ; R_{1} \geqslant p_{1} ;\right. \\
& R_{2} \leqslant \min \left(\frac{p_{b}-p_{2}-(1+\theta) R_{1}}{\theta}, \frac{p_{b}-\theta R_{1}-p_{1}}{(1+\theta)},\right. \\
& \left.\left.R_{1}-p_{1}+p_{2}\right)\right\} / m_{1} \\
= & \int_{p_{1}}^{\infty} \int_{p_{b} /(1+\theta)-r_{1}}^{\min \left(\left(p_{b}-p_{2}-(1+\theta) r_{1}\right) / \theta,\left(p_{b}-\theta r_{1}-p_{1}\right) /(1+\theta), r_{1}-p_{1}+p_{2}\right)} \\
& f_{R_{1}, R_{2}}\left(r_{1}, r_{2}\right) \mathrm{d} r_{1} \mathrm{~d} r_{2} / m_{1} .
\end{aligned}
$$


Following similar arguments, $\gamma_{12}, \gamma_{2 B}, \gamma_{21}, \gamma_{B 1}$ and $\gamma_{B 2}$ can be derived. The final expressions for the case $\theta>0$ are given below:

$$
\begin{aligned}
\gamma_{12}= & \int_{p_{1}}^{\infty} \int_{p_{2}}^{\min \left(\left(p_{b}-(1+\theta) r_{1}-p_{2}\right) / \theta,\left(p_{b}-\theta r_{1}-p_{1}\right) /(1+\theta), r_{1}-p_{1}+p_{2}\right)} \\
& f_{R_{1}, R_{2}}\left(r_{1}, r_{2}\right) \mathrm{d} r_{1} \mathrm{~d} r_{2} / m_{1} \\
\gamma_{2 B}= & \int_{p_{2}}^{\infty} \int_{\max \left(\left(p_{b}-p_{1}-(1+\theta) r_{2}\right) / \theta, p_{b} /(1+\theta)-r_{2}\right)}^{\min \left(\left(p_{b}-\theta r_{2}-p_{2}\right) /(1+\theta), r_{2}-p_{2}+p_{1}\right)} f_{R_{1}, R_{2}}\left(r_{1}, r_{2}\right) \mathrm{d} r_{2} \mathrm{~d} r_{1} / m_{2} \\
\gamma_{21}= & \int_{p_{2}}^{\infty} \int_{p_{1}}^{\min \left(\left(p_{b}-p_{1}-(1+\theta) r_{2}\right) / \theta,\left(p_{b}-\theta r_{2}-p_{2}\right) /(1+\theta), r_{2}-p_{2}+p_{1}\right)} \\
& f_{R_{1}, R_{2}}\left(r_{1}, r_{2}\right) \mathrm{d} r_{2} \mathrm{~d} r_{1} / m_{2} \\
\gamma_{B 1}= & \int_{p_{1}}^{\infty} \int_{\max \left(\left(p_{b}-\theta r_{1}-p_{1}\right) /(1+\theta),\left(p_{b}-(1+\theta) r_{1}-p_{2}\right) / \theta\right)}^{r_{1}-p_{1}+p_{2} ; r_{1} \geqslant p_{1}} \\
& f_{R_{1}, R_{2}}\left(r_{1}, r_{2}\right) \mathrm{d} r_{1} \mathrm{~d} r_{2} / m_{b} \\
\gamma_{B 2}= & \int_{p_{2}}^{\infty} \int_{\max \left(\left(-(1+\theta) r_{2}-p_{1}+p_{b}\right) / \theta,\left(p_{b}-\theta r_{2}-p_{2}\right) /(1+\theta)\right)}^{r_{2}-p_{2}+p_{1}} \\
& f_{R_{1}, R_{2}}\left(r_{1}, r_{2}\right) \mathrm{d} r_{2} \mathrm{~d} r_{1} / m_{b} .
\end{aligned}
$$

The final expressions for the case $\theta<0$ are given below:

$$
\begin{aligned}
\gamma_{12}= & \int_{p_{1}}^{\infty} \int_{\max \left(\left(p_{b}-(1+\theta) r_{1}-p_{2}\right) / \theta, p_{2}\right)}^{\min \left(\left(p_{b}-\theta r_{1}-p_{1}\right) /(1+\theta), r_{1}-p_{1}+p_{2}\right)} f_{R_{1}, R_{2}}\left(r_{1}, r_{2}\right) \mathrm{d} r_{1} \mathrm{~d} r_{2} / m_{1} \\
\gamma_{2 B}= & \int_{p_{2}}^{\infty} \int_{\frac{p_{b}}{1+\theta}-r_{2}}^{\min \left(\left(p_{b}-p_{1}-(1+\theta) r_{2}\right) / \theta,\left(p_{b}-\theta r_{2}-p_{2}\right) /(1+\theta), r_{2}-p_{2}+p_{1}\right)} \\
& \times f_{R_{1}, R_{2}}\left(r_{1}, r_{2}\right) \mathrm{d} r_{2} \mathrm{~d} r_{1} / m_{2} \\
\gamma_{21}= & \int_{p_{2}}^{\infty} \int_{\max \left(\left(p_{b}-p_{1}-(1+\theta) r_{2}\right) / \theta, p_{1}\right)}^{\min \left(\left(p_{b}-\theta r_{2}-p_{2}\right) /(1+\theta), r_{2}-p_{2}+p_{1}\right)} \\
& \times f_{R_{1}, R_{2}}\left(r_{1}, r_{2}\right) \mathrm{d} r_{2} \mathrm{~d} r_{1} / m_{2} \\
\gamma_{B 1}= & \int_{p_{1}}^{\infty} \int_{\left(p_{b}-\theta r_{1}-p_{1}\right) /(1+\theta)}^{\min \left(\left(p_{b}-(1+\theta) r_{1}-p_{2}\right) / \theta, r_{1}-p_{1}+p_{2}\right)} f_{R_{1}, R_{2}}\left(r_{1}, r_{2}\right) \mathrm{d} r_{1} \mathrm{~d} r_{2} / m_{b} \\
\gamma_{B 2}= & \int_{p_{2}}^{\infty} \int_{p_{b}-\theta r_{2}-p_{2} /(1+\theta)}^{\min \left(\left(-(1+\theta) r_{2}-p_{1}+p_{b} / \theta, r_{2}-p_{2}+p_{1}\right)\right.} f_{R_{1}, R_{2}}\left(r_{1}, r_{2}\right) \mathrm{d} r_{2} \mathrm{~d} r_{1} / m_{b} .
\end{aligned}
$$

Two types of products incur shortage: We first study the case when Products 1 and 2 run out of stock, and derive the expression for the switching probability from Product 1 to the Bundle.

$$
\begin{aligned}
\gamma_{1 B}^{-}= & \operatorname{Pr}\left\{R_{b} \geqslant p_{b} \mid R_{1}-p_{1} \geqslant R_{b}-p_{b} ; R_{1}-p_{1} \geqslant R_{2}-p_{2} ; R_{1} \geqslant p_{1}\right\} \\
= & \operatorname{Pr}\left\{(1+\theta)\left(R_{1}+R_{2}\right) \geqslant p_{b} ; R_{1}-p_{1} \geqslant(1+\theta)\left(R_{1}+R_{2}\right)-p_{b} ;\right. \\
& \left.R_{1}-p_{1} \geqslant R_{2}-p_{2} ; R_{1} \geqslant p_{1}\right\} / m_{1} \\
= & \operatorname{Pr}\left\{R_{2} \geqslant \frac{p_{b}}{1+\theta}-R_{1} ; \frac{p_{b}-\theta R_{1}-p_{1}}{1+\theta} \geqslant R_{2} ; R_{1}-p_{1}+p_{2}\right. \\
\geqslant & \left.R_{2} ; R_{1} \geqslant p_{1}\right\} / m_{1} \\
= & \operatorname{Pr}\left\{R_{2} \geqslant \frac{p_{b}}{1+\theta}-R_{1} ; R_{2} \leqslant \min \left(\frac{p_{b}-\theta R_{1}-p_{1}}{1+\theta},\right.\right. \\
& \left.\left.R_{1}-p_{1}+p_{2}\right) ; R_{1} \geqslant p_{1}\right\} / m_{1} \\
= & \int_{p_{1}}^{\infty} \int_{p_{b} /(1+\theta)-r_{1}}^{\min \left(\left(p_{b}-\theta r_{1}-p_{1}\right) /(1+\theta), r_{1}-p_{1}+p_{2}\right)} f_{R_{1}, R_{2}}\left(r_{1}, r_{2}\right) \mathrm{d} r_{1} \mathrm{~d} r_{2} / m_{1} .
\end{aligned}
$$

Similarly expressions for $\gamma_{2 B}^{-}, \gamma_{B 1}^{-}, \gamma_{21}^{-}, \gamma_{B 2}^{-}$, and $\gamma_{12}^{-}$can be derived. Final expressions for $\gamma_{2 B}^{-}, \gamma_{21}^{-}$, and $\gamma_{12}^{-}$are given below:

$$
\begin{aligned}
& \gamma_{2 B}^{-}=\int_{p_{2}}^{\infty} \int_{p_{b} /(1+\theta)-r_{2}}^{\min \left(\left(p_{b}-\theta r_{2}-p_{2}\right) /(1+\theta), r_{2}-p_{2}+p_{1}\right)} f_{R_{1}, R_{2}}\left(r_{1}, r_{2}\right) \mathrm{d} r_{2} \mathrm{~d} r_{1} / m_{2} \\
& \gamma_{21}^{-}=\int_{p_{2}}^{\infty} \int_{p_{1}}^{\min \left(\left(p_{b}-\theta r_{2}-p_{2}\right) /(1+\theta), r_{2}-p_{2}+p_{1}\right)} f_{R_{1}, R_{2}}\left(r_{1}, r_{2}\right) \mathrm{d} r_{2} \mathrm{~d} r_{1} / m_{2} \\
& \gamma_{12}^{-}=\int_{p_{1}}^{\infty} \int_{p_{2}}^{\min \left(\left(p_{b}-\theta r_{1}-p_{1}\right) /(1+\theta), r_{1}-p_{1}+p_{2}\right)} f_{R_{1}, R_{2}}\left(r_{1}, r_{2}\right) \mathrm{d} r_{1} \mathrm{~d} r_{2} / m_{1}
\end{aligned}
$$

Final expressions for $\gamma_{B 1}^{-}$and $\gamma_{B 2}^{-}$depend on the sign of $\theta$. These are given below for $\theta>0$ :

$$
\begin{aligned}
\gamma_{B 1}^{-}= & \int_{p_{1}}^{\infty} \int_{\max \left(\left(p_{b}-\theta r_{1}-p_{1}\right) /(1+\theta),\left(p_{b}-(1+\theta) r_{1}-p_{2}\right) / \theta\right)}^{\infty} \\
& \times f_{R_{1}, R_{2}}\left(r_{1}, r_{2}\right) \mathrm{d} r_{2} \mathrm{~d} r_{1} / m_{b} \\
\gamma_{B 2}^{-}= & \int_{p_{2}}^{\infty} \int_{\max \left(\left(-(1+\theta) r_{2}-p_{1}+p_{b}\right) / \theta,\left(p_{b}-\theta r_{2}-p_{2}\right) /(1+\theta)\right)}^{\infty} \\
& \times f_{R_{1}, R_{2}}\left(r_{1}, r_{2}\right) \mathrm{d} r_{2} \mathrm{~d} r_{1} / m_{b}
\end{aligned}
$$

and for $\theta<0$ :

$$
\begin{aligned}
& \gamma_{B 1}^{-}=\int_{p_{1}}^{\infty} \int_{\left(p_{b}-\theta r_{1}-p_{2}\right) /(1+\theta)}^{\left(p_{b}-(1+\theta) r_{1}-p_{2}\right) / \theta} f_{R_{1}, R_{2}}\left(r_{1}, r_{2}\right) \mathrm{d} r_{2} \mathrm{~d} r_{1} / m_{b} \\
& \gamma_{B 2}^{-}=\int_{p_{2}}^{\infty} \int_{\left(p_{b}-\theta r_{2}-p_{2}\right) /(1+\theta)}^{\left(-(1+\theta) r_{2}-p_{1}+p_{b}\right) / \theta} f_{R_{1}, R_{2}}\left(r_{1}, r_{2}\right) \mathrm{d} r_{2} \mathrm{~d} r_{1} / m_{b}
\end{aligned}
$$

\section{References}

Adams, W.J., Yellen, J.L., 1976. Commodity bundling and the burden of monopoly. Quarterly Journal of Economics 90, 475-498.

Ansari, A., Siddarth, S., Weinberg, C.B., 1996. Pricing a bundle of products and services: the case of nonprofits. Journal of Marketing Research 33, 86-93.

Bakos, Y., Brynjolfsson, E., 1999. Bundling information goods: pricing, profits, and efficiency. Management Science 45, 1613-1630.

Ben-Akiva, M., Gershenfeld, S., 1998. Multi-featured products and services: analysing pricing and bundling strategies. Journal of Forecasting 17, 175-196.

Bulut, Z., Gürler, Ü., Sen, A., 2009. Bundle pricing of inventories with stochastic demand. European Journal of Operational Research, in press, doi: 10.1016/j.ejor.2006.09.106.

Carbajo, J., de Meza, D., Seidmann, J.D., 1990. A strategic motivation for commodity bundling. The Journal of Industrial Economics 38, 283-298.

Choi, T.M., 2007. Pre-season stocking and pricing decisions for fashion retailers with multiple information updating. International Journal of Production Economics 106, 146-170.

Elmaghraby, W., Keskinocak, P., 2003. Dynamic pricing in the presence of inventory considerations: research overview, current practices, and future directions. Management Science 49, 1287-1309.

Ernst, R., Kouvelis, P., 1999. The effect of selling packaged goods on inventory decisions. Management Science 45 (8), 1142-1155.

Gallego, G., van Ryzin, G., 1997. A multiproduct dynamic pricing problem and its applications to network yield management. Operations Research 45 (1), 24-41.

Guiltinan, J.P., 1987. The price bundling of services: a normative framework. Journal of Marketing 51, 74-85.

Hanson, W.A., Martin, R.K., 1990. Optimal bundle pricing. Management Science 36 (2), 155-174.

Jedidi, K., Zhang, Z.J., 2002. Augmenting conjoint analysis to estimate consumer reservation price. Management Science 48 (10), $1350-1368$.

Jeuland, A., 1984. Comments on "Gaussian demand and commodity bundling. Journal of Business 57 (1), 230-235. 
Karakul, M., 2008. Joint pricing and procurement of fashion products in the existence of clearance markets. International Journal of Production Economics 114, 487-506.

Long, J.B., 1984. Comments on Gaussian demand and commodity bundling. Journal of Business 57 (1), 235-246.

McAffe, R.P., McMillan, J., Whinston, M.D., 1989. Multiproduct monopoly, commodity bundling, and correlation of values. Quarterly Journal of Economics 104, 371-384.

Nalebuff, B. 2003. Bundling, Tying, and Portfolio Effect. Part I: conceptual Issues. Technical Report, Department of Trade and Industry, London, England.

Netessine, S., Savin, S., Xiao, W., 2004. Dynamic revenue management through cross-selling in e-commerce retailing. Operations Research 54 (5), 893-913.

Salinger, M.A., 1995. A graphical analysis of bundling. Journal of Business 68 (1), 85-98.

Sandholm, T., Levine, D., Concordia, M., Martyn, P., Hughes, R., Jacobs, J., Begg, D., 2006. Changing the game in strategic sourcing at Procter \&
Gamble: expressive competition enabled by optimization. Interfaces 36, 55-68.

Schmalensee, R., 1984. Gaussian demand and commodity bundling Journal of Business 57 (1), 211-230.

Șen, A., 2008. The U.S. fashion industry: a supply chain review. International Journal of Production Economics 114, 571-593.

Stigler, G.J., 1963. United States vs. Loew's Inc.: a note on block booking Supreme Court Review 152-157.

Stremersch, S., Tellis, G.J., 2002. Strategic bundling of products and prices: a new synthesis for marketing. Journal of Marketing 66, 55-72.

Talluri, T., van Ryzin, G., 2004. The Theory and Practice of Revenue Management. Kluwer Academic Publishers, Dordrecht.

Venkatesh, R., Kamakura, W., 2003. Optimal bundling and pricing under a monopoly: contrasting complements and substitutes from independently valued products. Journal of Business 76 (2), 211-231. 